\title{
Zur de Broglie'schen Theorie der Elementarteilchen
}

\author{
Von Helmut Hönl * und Hermann Boerner ** \\ (Z. Naturforschg. 5 a, 353-366 [1950]; eingegangen am 21. März 1950)
}

\begin{abstract}
L. de Broglie hat eine einfache Konstruktionsvorschrift angegeben, um von der Wellengleichung für das Elektron zu Teilchen mit höherem Spin aufzusteigen (méthode de fusion). Um Aussagen über die individuellen Eigenschaften von Teilchen mit höherem Spin zu erhalten, ist es erforderlich, die nach der de Broglieschen Vorschrift konstruierten Matrixsysteme vollständig auszureduzieren. Diese Aufgabe wird im mathematischen Teil der Arbeit (II bis VI) für die Fusionsstufe $N=3$ vollständig durchgeführt, und zwar ohne das Hilfsmittel der Darstellungstheorie der Drehgruppe im $R_{5}(\mathrm{~L} \mathrm{u} \mathrm{bank} \mathrm{ki),} \mathrm{nachdem} \mathrm{die} \mathrm{analoge} \mathrm{Aufgabe} \mathrm{für} N=2$ schon vor einigen Jahren durch $\mathrm{Kemmer}$ in seiner Mesonentheorie (ausgehend von den Duffinschen Vertauschungsrelationen) behandelt worden ist. Es zeigt sich, daß sich die ausreduzierten Matrizen $\gamma_{\mu}(\mu=1, \ldots, 4)$ in den Wellengleichungen der Fusionsstufe $N=3$ in Kronecker-Produkte $\gamma_{\mu}=\Gamma_{\mu} \times \alpha_{\mu}$ zerlegen lassen, wobei die $\alpha_{\mu}$ gewöhnliche Dirac-Matrizen sind und die $\Gamma_{\mu}$ sich durch 5-, 4- und 1-reihige Matrizen darstellen lassen (V); die zugehörigen ausreduzierten Wellengleichungen (VI) enthalten also 5.4, $4 \cdot 4$ bzw. $1 \cdot 4$ Komponenten. Die Diskussion ergibt, daß die 4-komponentige Wellengleichung mit der Dirac-Gleichung identisch ist (also nichts Neues liefert), während die Wellengleichungen mit $4 \cdot 4$ bzw. $5 \cdot 4$ Komponenten je 3 Teilchen mit verschiedenen individuellen Eigenschaften enthalten. Die auftretenden Spinwerte sind $\hbar / 2$ und $\hbar / 2$, die zu einer Darstellung gehörenden Massenwerte stehen im Verbältnis 1:3 (VII). Die Berechnung des magnetischen Moments eines Teilchens der Stufe 3 wird für einen Spezialfall durchgeführt (VIII). Eine Zusammenstellung der Massen- und Spinwerte sowie der magnetischen $g$-Werte der Teilchen findet sich im Schlußabsatz (IX). Unter den Teilchen der Stufe 3 befindet sich keines, das ohne zusätzliche Annahmen dem Proton zugeordnet werden könnte.
\end{abstract}

I.

$\ddot{U}$ ber die Struktur der Elementarteilchen wissen wir heute noch wenig. Das einzige Teilchen, von dem wir begründeterweise behaupten können, daß wir seine Struktur in einem weiten Bereiche beherrschen, ist das Elektron (bzw. auch Positron). D i r a c hat bekanntlich eine relativistisch invariante Wellengleichung erster Ordnung aufgestellt, welche sowohl Spin wie magnetisches Moment des Elektrons enthält und ferner die Erscheinungen der Paarerzeugung und Paarvernichtung zu beschreiben gestattet. Aber schon die Wellengleichung des Protons ist uns unbekannt. Es ist nicht sehr wahrscheinlich, daß die Wellengleichung des Protons trotz des gleichen Spins $\hbar / 2$ mit derjenigen für das Elektron übereinstimmt, da das magnetische Moment des Protons demjenigen des Elektrons nicht analog ist ${ }^{1}$. Man kann selbst daran zweifeln, ob sich für das Proton unabhängig vom Neutron eine Wellengleichung formulieren läßt, da

* Ebnet bei Freiburg i. Br., Hauptstr. 64.

1 Das magnetische Moment des Protons beträgt etwa 2,79 Kernmagnetonen. (Genauester heute bekannter Wert: $\mu=2,7926 \pm 0,0006$ K.M. Vgl. Millman u. Kusch, Physic. Rev. 56, 303 [1939].)

2 A. Proca, J. Physique Radium 7, 347 [1936]; 9, 61 [1938].
Proton und Neutron nach den Erfahrungen über die intranuclearen Wechselwirkungen als Modifikationen desselben Gebildes aufzufassen sind. Endlich haben Proca ${ }^{2}$ und $\mathrm{Kemmer}{ }^{3}$ Wellengleichungen für Mesonen aufgestellt, welche möglicherweise zutreffen, wenigstens soweit es sich um Mesonen vom Spin 0 und $\hbar$ handelt.

Fragt man, welche Möglichkeiten die gegenwärtige Theorie besitzt, um zu Wellengleichungen für Teilchen mit beliebigen halb- oder ganzzahligen Spinwerten und mit individuellen Eigenschaften, welche von denjenigen des Elektrons verschieden sind, zu gelangen, so bieten sich im Prinzip zwei verschiedene Wege dar. Man kann entweder formal die Diracsche Wellengleichung so zu erweitern suchen, daß dabei unter Festhaltung an der Diracschen Form und der Forderung der relativistischen Invarianz beliebige halb- oder ganzzahlige Spinwerte herauskommen; dieser Weg ist zuerst von $\mathrm{de} \mathrm{B} \mathrm{rog} \mathrm{li} \mathrm{e}^{4}$ beschritten

** Gießen, Fichtestr. 13.

3 N. Ke m mer, Proc. Roy. Soc. [London], Ser. A 173, 91 [1940].

4 L. de B r o g li e, Actualités scient., Hermann, Paris, Nr. 181 [1934], 411 [1936]. Zusammenfassend: Théorie générale des particules à spin (méthode de fusion), Gauthiers-Villars, Paris 1943. 
worden und hat seither zu einer Reihe von Untersuchungen $^{5}$ über Dirac-ähnliche Wellengleichungen Anlaß gegeben. Andererseits ist von $\mathrm{Bopp} \mathrm{p}^{6}$ ein zweiter Weg eingeschlagen worden, der zunächst darauf abzielte, das Maxwellsche Vakuumfeld in allgemeiner Weise derart zu modifizieren, daß darin Teilchen als Feldsingularitäten im Sinne der Mie-Bornschen Theorie der Materie möglich erscheinen. Im Fortgang dieser Untersuchungen erhebt sich dann insbesondere die Frage nach der Mechanik der Feldsingularitäten in einer gewissen, über die Lorentzsche Elektronentheorie hinausgehenden Näherung und nach der Übertragung des zugehörigen kanonischen Formalismus dieser „Feldmechanik“ in die Quantentheorie ${ }^{6 \text { a }}$. Es zeigt sich dann, daß die „klassischen“ Teilchen bereits das Spinphänomen als eine Art Zitterbewegung enthalten und daß bei der Úbertragung gewisser Poisson-Klammern als Funktionen der Spinvariabeln in die Quantenmechanik, auf welche schon W e s s e ${ }^{7}$ hingewiesen hat, sich der Übergang zu Dirac-ähnlichen Wellengleichungen zwangsläufig und willkürfrei vollziehen läßt ${ }^{8}$. Es ist gewiß befriedigend, wenn sich dabei herausstellt, daß sich die Formulierung der Wellengleichung und der Vertauschungsrelationen für die erweiterten Dirac-ähnlichen Matrizen in genauer Úbereinstimmung mit dem de B r o g li e schen Verfahren ergibt, wodurch dieses erst auf eine breitere theoretische Grundlage gestellt wird.

Diese Übereinstimmung ist für uns der hauptsächlichste Gesichtspunkt, wenn wir das de Brogliesche Verfahren vorläufig bedenkenlos unserer Untersuchung zugrunde legen.

Wir schreiben die Diracsche Wellengleichung in der symmetrischen Form ${ }^{8 \mathrm{a}}$ :

5 F. J. Belinfante, Physica 6, 849, 887 [1939]; H. A. Kramers, F. J. Belinf ante u. J. K. Lubański, Physica 8, 597 [1941]; H. B habha, Rev. mod. Physics 17, 200 [1945]; Proc. Indian Acad. Sci. 21, 265 [1945]; B. S. Madhavarao, V. R. Thiruvenkatachar u. K. Venkatachaliengar, Proc. Roy. Soc. [London], Ser. A 187, 385 [1946]; vgl. auch B. S. Madhavara o, Proc. Indian Acad. Sci. 15, 139 [1942]. - Neuerdings: H. B h a b ha, Rev. mod. Physics 21, 451 [1949] (Einstein-Heft); F. B o p p u. F. L. B a u e r, Z. Naturforschg. 4 a, 611 [1949]; F. L. B a u e r, ebenda 4 a, 720 [1949] (Notiz).

6 F. B opp, Ann. Physik 42, 572 [1943]; 43, 565 [1943], Z. Naturforschg. 1, 53 [1946]; Z. Physik 125, 615 [1949].

6a F. B opp, Z. Naturforschg. 1, 146 [1948].

7 W. We s s el, Z. Naturforschg. 1, 622 [1946].

8 F. B op p, Z. Naturforschg. 3a, 564 [1948]; F. B o p p u. F. L. B a u er 5 .

8 a Griechische Indizes laufen stets von 1 bis 4; Summation über gleiche Indizes.

$$
\begin{gathered}
\left(a_{\mu} \partial \mu+\varkappa\right) \psi=0, \\
\%=m_{0} \mathrm{c} / \hbar, \quad \partial \mu=\partial / \partial x_{\mu}, \\
\epsilon_{\mu} c_{\nu}+c_{\nu} c_{\mu}=2 \delta_{\mu \nu},
\end{gathered}
$$

$\left(x_{\mu}\right.$ Raum-Zeitkoordinaten, von der Einführung eines Viererpotentials werde zunächst abgesehen). Die Wellengleichung für Teilchen mit beliebigem Spin sei analog zu (I. 1)

$$
\left(\gamma_{\mu} \partial \mu+N \varkappa\right) \psi=0 ;
$$

dann lautet die de Brogliesche Vorschrift zur Bildung $\operatorname{der} \gamma_{\mu}$ :

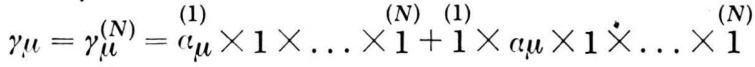

$$
\begin{aligned}
& +\ldots+\stackrel{(1)}{1} \times 1 \times \ldots \times \stackrel{(N)}{a_{\mu}},
\end{aligned}
$$

d.h. $\gamma_{\mu}$ gleich der Summe von $N$-fachen „KroneckerProdukten " der $\alpha_{\mu}$ mit vierreihigen Einsmatrizen $\left(N\right.$ heiße „Stufe der Verschmelzung“) ${ }^{9}$. Es tritt dann zu dem Bahnimpulsmoment der Bewegung ein $\mathrm{Zu}$ satzimpulsmoment (Spin) mit den Komponenten

$$
\begin{aligned}
S_{i k} & =\frac{1}{4 i}\left(\gamma_{j} \gamma_{k}-\gamma_{k} \gamma_{j}\right) \cdot \hbar, \\
((j, k) & =(1,2),(2,3),(3,1)),
\end{aligned}
$$

welches zusammen mit dem Bahnimpulsmoment eine Konstante der Bewegung ausmacht. Die Eigenwerte von (I.5) sind:

$$
(0,1, \ldots N / 2) \hbar \text { für geradzahliges } N \text {, }
$$

$(1 / 2,3 / 2, \ldots N / 2) \hbar$ für ungeradzahliges $N$,

maximal also jeweils $N \hbar / 2^{10}$. - Die Spinkomponenten sind stets mit $\gamma_{4}$ vertauschbar; allgemeiner gilt folgende Vertauschungsrelation ${ }^{11}$, die man nach (I. 2)

9 Das Kronecker-Produkt $A \times B$ zweier Matrizen $A=\left\{a_{i k}\right\}$ und $B=\left\{b_{i k}\right\}$ (nicht notwendig gleicher Zeilenzahl) ist bekanntlich die Matrix $\left\{a_{i h} b_{j k}\right\}$, deren Zeilenindex die Wertepaare $(i, i)$, deren Spaltenindex die Zahlenpaare $(h, k)$ durchläuft. Die Verallgemeinerung für mehr als 2 Faktoren liegt auf der Hand. Das Matrixprodukt zweier gleichartiger Kronecker-Produkte $A_{1} \times A_{2}$ $\times \ldots \times A_{m}$ und $B_{1} \times B_{2} \times \ldots \times B_{m}$ (,gleichartig“" heißt: für jedes $i$ stimmen $A_{i}$ und $B_{i}$ in der Zeilenzahl überein) ist einfach $A_{1} B_{1} \times A_{2} B_{2} \times \ldots \times A_{m} B_{m}$.

10 In historischer Hinsicht sei bemerkt, daß das de Brogliesche Verfahren aus dem Versuche hervorging, das Lichtquant aufzufassen als "Verschmelzung“" von Neutrino und Antineutrino, welche ihrerseits einer Diracschen Wellengleichung (mit $\varkappa=0$ ) genügen (Neutrinotheorie des Lichts). Mesonen wären gleichsam hiernach "schweres Licht".

11 J. K. Lubański, Physica 9, 310, 325 [1942]. Vgl. auch B. S. Madhavarao u. Mitarbb. ${ }^{5}$. - Eckige Klammern bedeuten Kommutatoren: $[a, b]=a b-b a$. 
und (I.4) leicht bestätigt:

$$
\left[\left[\gamma_{\mu}, \gamma_{\nu}\right] \gamma_{\varrho}\right]=4\left(\gamma_{\mu} \delta_{\nu \varrho}-\gamma_{\nu} \delta_{\mu \varrho}\right) .
$$

Die Vertauschungsrelationen (I.6) sind andererseits von $\mathrm{B}$ o $\mathrm{p} \mathrm{p}^{8}$ aus den entsprechenden Relationen für die Poisson-Klammern der Feldmechanik korrespondenzmäßig erschlossen worden.

Die Anwendung der Wellengleichung (I. 3) in Verbindung mit den Vertauschungsrelationen (I.6) auf Elementarteilchen ergibt sich im Zusammenhang mit der Darstellungstheorie der Matrizen $\gamma_{\mu}$. Man wird erwarten dürfen, daß den einzelnen irreduziblen Matrixsystemen $\gamma_{\mu}$ einzelne Elementarteilchen oder Gruppen von solchen entsprechen werden. Die irreduziblen Bestandteile der von de Broglie gegebenen (nicht völlig allgemeinen ${ }^{12}$ ) Darstellungen (I. 4) sind für $N=2$ von $\mathrm{Kemmer} \mathrm{men}^{3}$ angegeben und seiner Mesonentheorie zugrunde gelegt worden. Für beliebiges $N$ ist die Frage nach den möglichen Darstellungen ausführlich zuerst von $\mathrm{Lub}$ a ński ${ }^{9}$ und neuerdings von Bopp und F. L. B a u e ${ }^{5}$ behandelt worden durch Zurückführung auf die Darstellungstheorie der Lieschen Ringe der Drehgruppen. Wir beschränken uns hier auf die Fälle $N=2$ und $N=3$. Dabei liegt das Hauptgewicht der Untersuchung in methodischer Hinsicht darauf, eine "independente", d. h. den Apparat der Darstellungstheorie der Drehgruppe nicht benutzende und dennoch - im Gegensatz z. B. zu Kemmer für $N=2$ - vollständig ausgeführte Herleitung der Ausreduktion in diesen Fällen zu geben, mit numerischer Angabe von Matrizen und Wellengleichungen. Eine solche Behandlungsweise mag vor allem deswegen erwünscht sein, weil die sich hierbei ergebenden Gesichtspunkte für die Ermittlung spezieller Darstellungen (Matrizen) sich auf allgemeinere Fälle übertragen lassen (z. B. Übergang von irgendeinem geradzahligen $N$ zum nächsthöheren ungeradzahligen).

Die vorliegende Untersuchung gliedert sich in einen vorwiegend mathematischen, die Algebra der Matrix-

12 Die Vertauschungsrelationen (I.6) lassen noch verschiedene Vorzeichen-Kombinationen in dem Aggregat (I. 4) zu. Vgl. H. A. Kramers, F. J. Belinfante u. J. K. Lubański 5 .

13 Es mag uns erlaubt sein zu bemerken, daß der mathematische Teil schon längere Zeit zum größten Teile abgeschlossen vorlag, jedoch in Abschn. VI (Wellengleichungen) durch ein von Hrn. F. L. B a u er freundlich zur Verfügung gestelltes Manuskript später eine wesentliche Förderung erfuhr. Übrigens weichen die von F. L. Bauer kürzlich mitgeteilten ausreduzierten Wellengleichungen ${ }^{5}$ für $N=3$ von den unserc . nur in der .Bezeichnung etwas ab.
Systeme und ihre Darstellungen enthaltenden Teil (Abschn. II bis VI) und in einen physikalischen Teil, welcher der Diskussion der ausreduzierten Wellengleichungen und der sich ergebenden magnetischen Momente der Elementarteilchen gewidmet ist (Abschnitte VII bis IX) ${ }^{13}$.

\section{Algebra der $a$ - und $\eta$-Systeme}

Die 4 Diracschen Größen $\alpha_{\mu}$ genügen den Relationen

$$
a_{\mu} c_{v}+a_{\nu} c_{\mu}=2 \delta_{\mu v} .
$$

Sie erzeugen eine Algebra von 16 Dimensionen, für die z. B. die 16 Größen

$1, c_{\mu}, a_{\mu} c_{v}, a_{\mu} c_{v}, c_{Q}, a_{1} a_{2} c_{3} c_{4}(\mu<v<\varrho)$

eine Basis bilden und die eine irreduzible Darstellung vom Grad 4 besitzt, etwa durch die Matrizen

$$
\begin{array}{ll}
a_{1} \sim\left(\begin{array}{rrrr}
0 & 0 & 0 & 1 \\
0 & 0 & 1 & 0 \\
0 & 1 & 0 & 0 \\
1 & 0 & 0 & 0
\end{array}\right), & a_{2} \sim\left(\begin{array}{rrrr}
0 & 0 & 0 & -\mathrm{i} \\
0 & 0 & \mathrm{i} & 0 \\
0 & -\mathrm{i} & 0 & 0 \\
\mathrm{i} & 0 & 0 & 0
\end{array}\right), \\
a_{3} \sim\left(\begin{array}{rrrr}
0 & 0 & 1 & 0 \\
0 & 0 & 0 & -1 \\
1 & 0 & 0 & 0 \\
0 & -1 & 0 & 0
\end{array}\right), & \iota_{4} \sim\left(\begin{array}{rrrr}
1 & 0 & 0 & 0 \\
0 & 1 & 0 & 0 \\
0 & 0 & -1 & 0 \\
0 & 0 & 0-1
\end{array}\right) ;
\end{array}
$$

wegen $4^{2}=16$ ist dies die einzige ${ }^{14}$. Jede andere 4-reihige Darstellung ist daher zu dieser äquivalent, insbesondere alle Darstellungen, die man aus (II. 3) durch Permutation der 4 Indizes erhalten kann; eine Tatsache, die man die "Austauschbarkeit" der $\alpha$-Matrizen nennen kann. Wir nennen jedes System von 4 Größen, die wie die $\alpha_{\mu}$ antikommutativ sind und die Quadrate 1 haben und zwischen denen keine weitere von diesen unabhängige Relation besteht (so daß die von ihnen erzeugte Algebra zur $\alpha$-Algebra isomorph ist), ein $\alpha$-System.

In der Kemmerschen Theorie kommen 4 Größen $\eta_{\varphi}$ vor, die den Relationen

$$
\eta_{\mu}^{2}=1, \quad \eta_{\mu} \eta_{v}=\eta_{v}, \eta_{\mu}
$$

14 Für die „halbeinfachen“ Algebren, d. h. die „vollreduziblen“, deren Darstellungen vollständig in irreduzible zerfallen, gelten folgende im Text benutzten Sätze: 1. Die Dimension der Algebra ist die Quadratsumme der Grade der verschiedenen (inäquivalenten) irreduziblen Darstellungen; 2. Eine Darstellung ist dann und nur dann treu (d. h. umkehrbar eindeutiges Bild der Algebra), wenn bei ihrer Zerlegung jede irreduzible mindestens einmal vorkommt. Für Halbeinfachheit ist die Existenz einer treuen Darstellung hinreichend, die vollständig zerfällt. Die $\alpha$-Algebra ist nicht nur halbeinfach, sondern ,einfach“, da sie eine einzige (treue) irreduzible Darstellung besitzt. 
genügen. Sie erzeugen ebenfalls eine Algebra von 16 Dimensionen, für die z. B. die Größen

$1, \eta_{\mu}, \eta_{\mu} \eta_{v}, \eta_{\mu} \eta_{v} \eta_{\varrho}, \eta_{1} \eta_{2} \eta_{3} \eta_{4}(\mu<v<\varrho)$

eine Basis bilden. Als kommutative Algebra besitzt sie nur eindimensionale Darstellungen, und jedes $\eta_{\mu}$ wird durch \pm 1 dargestellt. Es gibt 16 verschiedene Möglichkeiten, die Vorzeichen + und - auf die 4 Größen $\mathrm{zu}$ verteilen, und somit 16 verschiedene irreduzible Darstellungen, wie es sein muß. Wir schreiben sie in einer bestimmten Reihenfolge hin, die durch das folgende Schema festgelegt ist, dessen 16 Spalten die 16 Darstellungen bedeuten; statt \pm 1 ist einfach + oder - geschrieben:

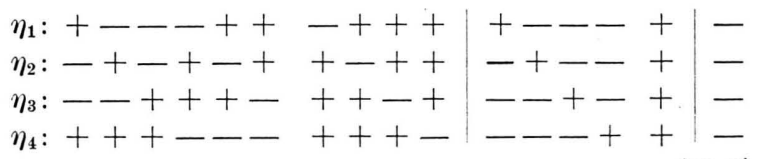

(II. 6)

Unter $\eta_{\mu}$ sollen im folgenden vier 16-reihige Matrizen verstanden werden, in denen die 16 Darstellungen in dieser Reihenfolge aneinandergereiht sind, also 4 Diagonalmatrizen aus \pm 1 , für welche die Reihenfolge der Vorzeichen durch die 4 Zeilen von (II.6) gegeben ist. 4 kommutative Größen mit den Quadraten 1 , zwischen denen keine weitere Relation besteht, heißen ein $\eta$-System.

\section{D e r F all $N=2$}

$x_{\mu}$ und $y_{\mu}(\mu=1, \ldots, 4)$ seien zwei vertauschbare $\alpha$-Systeme, also 8 Größen, zwischen denen die Relationen

$$
\begin{gathered}
x_{\mu} x_{v}+x_{v} x_{\mu}=y_{\mu} y_{\nu}+y_{\nu} y_{\mu}=2 \delta_{\mu \nu}, \\
x_{\mu} y_{v}-y_{v} x_{\mu}=0
\end{gathered}
$$

bestehen. Die von diesen Größen erzeugte „x-yAlgebra" hat die Dimension $16^{2}=256$, denn die Produkte aus einem Basiselement der $x$-Algebra mit einem solchen der $y$-Algebra sind linear unabhängig und spannen die ganze $x$-y-Algebra auf.

Eine Teilalgebra dieser Algebra ist die von den Größen $\beta_{\mu}=x_{\mu}+y_{\mu}$ erzeugte , $\beta$-Algebra“. Die $\beta_{\mu}$ erfüllen, wie man sich durch Einsetzen und Berücksichtigung von (III. 1) überzeugt, die Vertauschungsrelationen (I.6) und die $\beta$-Algebra ist zur Kemmer- schen (I. 4) mit $N=2$ isomorph, wie aus dem folgenden hervorgeht.

Die Größen $z_{\mu}=x_{\mu} y_{\mu}$ bilden ein $\eta$-System, das mit den $x_{\mu}$ und $y_{\mu}$ durch die Relationen

$$
\begin{gathered}
x_{\mu} y_{\mu}=y_{\mu} x_{\mu}=z_{\mu}, x_{\mu} z_{\mu}=z_{\mu} x_{\mu}=y_{\mu} \\
y_{\mu} z_{\mu}=z_{\mu} y_{\mu}=x_{\mu}, \\
x_{\mu} z_{v}+z_{v} x_{\mu}=y_{\mu} z_{v}+z_{v} y_{\mu}=0(\mu \neq v)
\end{gathered}
$$

verknüpft ist, die alle mit Leichtigkeit aus (III. 1) folgen. Die von ihnen erzeugte Teilalgebra ist wegen $\frac{1}{2} \beta^{2} \mu-1=z_{\mu}$ in der $\beta$-Algebra enthalten.

Nun kennen wir eine treue Darstellung der $x-y$ Algebra vom Grad 16, nämlich

$$
x_{\mu}=a_{\mu} \times 1, \quad y_{\mu}=1 \times a_{\mu} .
$$

Weil sie treu ist und weil $16^{2}=256$ ist, ist sie irreduzibel und die einzige irreduzible Darstellung. Sie enthält eine treue Darstellung der $\beta$-Algebra; wenn wir diese in irreduzible zerfällen können, so ist damit gezeigt: erstens daß die $\beta$-Algebra halbeinfach ist, zweitens daß uns hiermit alle ihre irreduziblen Darstellungen vorliegen ${ }^{14}$.

Unsere 16-reihige Darstellung enthält weiter eine treue Darstellung der $z$-Algebra, die wir sofort ausreduzieren können. Sie ist ja zur $\eta$-Algebra des vorigen Abschnitts isomorph, und wegen der Treue und wegen der Dimension muß offenbar jede von deren 16 irreduziblen Darstellungen genau einmal vorkommen. Nehmen wir also $z_{\mu}=\eta_{\mu}$ an, wo $\eta_{\mu}$ die durch (II.6) gegebenen Diagonalmatrizen sind, und sehen wir zu, wie dann die $x_{\mu}$ und die $y_{\mu}$ aussehen.

(II. 2) verlangt zunächst, daß $x_{\mu}$ mit $z_{\mu}$ vertauschbar und mit $z_{v}(\nu \neq \mu)$ antikommutativ ist. Die Vertauschbarkeit einer Matrix $\left\{a_{i k}\right\}$ mit einer Diagonalmatrix $\left\{c_{i}\right\}$ erfordert für jedes $(i, k) c_{i} a_{i k}=a_{i k} c_{k}$, also entweder $c_{i}=c_{k}$ oder $a_{i k}=0$; Antikommutativität entsprechend: $c_{i}=-c_{k}$ oder $a_{i k}=0$. Daher kann man an dem Schema (II.6) sofort ablesen, an welchen Stellen $(i, k)$ der $x_{\mu}$ darstellenden Matrix nur von Null verschiedene Zahlen stehen können: es muß die $i$-te und die $k$-te Spalte von (II. 6) in der $\mu$-ten Zeile das gleiche Zeichen, in den drei übrigen Zeilen entgegengesetzte Zeichen zeigen. Das sind die folgenden Stellen $(i, k)$ :

$$
\begin{array}{rr|ll}
\mu=1: & (1,10),(5,9),(6,8),(11,15) & (2,13),(3,12),(4,14),(7,16) \\
\mu=2: & (2,10),(4,9),(6,7),(12,15) & (1,13),(3,11),(5,14),(8,16) \\
\mu=3: & (3,10),(4,8),(5,7),(13,15) & (1,12),(2,11),(6,14),(9,16) \\
\mu=4: & (1,7),(2,8),(3,9),(14,15) & (4,11),(5,12),(6,13),(10,16)
\end{array}
$$


und jeweils die an der Hauptdiagonale gespiegelte Stelle $(k, i)$; in jeder Zeile und in jeder Spalte ist es genau eine Stelle.

Dasselbe gilt für $y_{\mu}$; wegen (III. 2) $x_{\mu} z_{\mu}=y_{\mu}$ stimmt dabei $y_{\mu}$ an den Stellen, die einem + in (II. 6 ) entsprechen, mit $x_{\mu}$ überein [diese Stellen machen die linke Hälfte des Schemas (III. 3) aus], an den andern hat es entgegengesetztes Vorzeichen.

Durch die Relationen (III.1) werden die für die fraglichen Matrixelemente $a_{i k}$ von $x_{\mu}$ möglichen Zahlenwerte weiter eingeschränkt. $x^{2} \mu=1$ verlangt $a_{i k} a_{k i}=1$, woraus [für die in (III.3) angegebenen $(i, k)] a_{i k} \neq 0$ folgt. Weiter erfordert die Antikommutativität der $x_{\mu}$ untereinander eine große Zahl von Beziehungen zwischen den Matrixelementen. Hat man dies alles erfüllt, so folgen die entsprechenden Relationen für die $y_{\mu}$ und die Vertauschbarkeit von $x_{\mu}$ und $y_{\mu}$ von selbst. Die Erfüllbarkeit all dieser Be-

$$
x_{1}=
$$

$\left(\begin{array}{rrrrrrrrrr|rrrrr|r}0 & 0 & 0 & 0 & 0 & 0 & 0 & 0 & 0 & 1 & 0 & 0 & 0 & 0 & 0 & 0 \\ 0 & 0 & 0 & 0 & 0 & 0 & 0 & 0 & 0 & 0 & 0 & 0 & 1 & 0 & 0 & 0 \\ 0 & 0 & 0 & 0 & 0 & 0 & 0 & 0 & 0 & 0 & 0 & -1 & 0 & 0 & 0 & 0 \\ 0 & 0 & 0 & 0 & 0 & 0 & 0 & 0 & 0 & 0 & 0 & 0 & 0 & -1 & 0 & 0 \\ 0 & 0 & 0 & 0 & 0 & 0 & 0 & 0 & -1 & 0 & 0 & 0 & 0 & 0 & 0 & 0 \\ 0 & 0 & 0 & 0 & 0 & 0 & 0 & 1 & 0 & 0 & 0 & 0 & 0 & 0 & 0 & 0 \\ 0 & 0 & 0 & 0 & 0 & 0 & 0 & 0 & 0 & 0 & 0 & 0 & 0 & 0 & 0 & 1 \\ 0 & 0 & 0 & 0 & 0 & 1 & 0 & 0 & 0 & 0 & 0 & 0 & 0 & 0 & 0 & 0 \\ 0 & 0 & 0 & 0 & -1 & 0 & 0 & 0 & 0 & 0 & 0 & 0 & 0 & 0 & 0 & 0 \\ 1 & 0 & 0 & 0 & 0 & 0 & 0 & 0 & 0 & 0 & 0 & 0 & 0 & 0 & 0 & 0 \\ \hline 0 & 0 & 0 & 0 & 0 & 0 & 0 & 0 & 0 & 0 & 0 & 0 & 0 & 0 & 1 & 0 \\ 0 & 0 & -1 & 0 & 0 & 0 & 0 & 0 & 0 & 0 & 0 & 0 & 0 & 0 & 0 & 0 \\ 0 & 1 & 0 & 0 & 0 & 0 & 0 & 0 & 0 & 0 & 0 & 0 & 0 & 0 & 0 & 0 \\ 0 & 0 & 0 & -1 & 0 & 0 & 0 & 0 & 0 & 0 & 0 & 0 & 0 & 0 & 0 & 0 \\ 0 & 0 & 0 & 0 & 0 & 0 & 0 & 0 & 0 & 0 & 1 & 0 & 0 & 0 & 0 & 0 \\ \hline 0 & 0 & 0 & 0 & 0 & 0 & 1 & 0 & 0 & 0 & 0 & 0 & 0 & 0 & 0 & 0\end{array}\right)$

$x_{2}=$

$\left(\begin{array}{rrrrrrrrrr|rrrrr|r}0 & 0 & 0 & 0 & 0 & 0 & 0 & 0 & 0 & 0 & 0 & 0 & -1 & 0 & 0 & 0 \\ 0 & 0 & 0 & 0 & 0 & 0 & 0 & 0 & 0 & 1 & 0 & 0 & 0 & 0 & 0 & 0 \\ 0 & 0 & 0 & 0 & 0 & 0 & 0 & 0 & 0 & 0 & 1 & 0 & 0 & 0 & 0 & 0 \\ 0 & 0 & 0 & 0 & 0 & 0 & 0 & 0 & 1 & 0 & 0 & 0 & 0 & 0 & 0 & 0 \\ 0 & 0 & 0 & 0 & 0 & 0 & 0 & 0 & 0 & 0 & 0 & 0 & 0 & -1 & 0 & 0 \\ 0 & 0 & 0 & 0 & 0 & 0 & -1 & 0 & 0 & 0 & 0 & 0 & 0 & 0 & 0 & 0 \\ 0 & 0 & 0 & 0 & 0 & -1 & 0 & 0 & 0 & 0 & 0 & 0 & 0 & 0 & 0 & 0 \\ 0 & 0 & 0 & 0 & 0 & 0 & 0 & 0 & 0 & 0 & 0 & 0 & 0 & 0 & 0 & 1 \\ 0 & 0 & 0 & 1 & 0 & 0 & 0 & 0 & 0 & 0 & 0 & 0 & 0 & 0 & 0 & 0 \\ 0 & 1 & 0 & 0 & 0 & 0 & 0 & 0 & 0 & 0 & 0 & 0 & 0 & 0 & 0 & 0 \\ \hline 0 & 0 & 1 & 0 & 0 & 0 & 0 & 0 & 0 & 0 & 0 & 0 & 0 & 0 & 0 & 0 \\ 0 & 0 & 0 & 0 & 0 & 0 & 0 & 0 & 0 & 0 & 0 & 0 & 0 & 0 & 1 & 0 \\ -1 & 0 & 0 & 0 & 0 & 0 & 0 & 0 & 0 & 0 & 0 & 0 & 0 & 0 & 0 & 0 \\ 0 & 0 & 0 & 0 & -1 & 0 & 0 & 0 & 0 & 0 & 0 & 0 & 0 & 0 & 0 & 0 \\ 0 & 0 & 0 & 0 & 0 & 0 & 0 & 0 & 0 & 0 & 0 & 1 & 0 & 0 & 0 & 0 \\ \hline 0 & 0 & 0 & 0 & 0 & 0 & 0 & 1 & 0 & 0 & 0 & 0 & 0 & 0 & 0 & 0\end{array}\right)$

dingungen ist natürlich durch das, was wir wissen, gewährleistet. Man kann sie in der Tat auf verschiedene Arten erfüllen. Wir geben hier eine einfache Lösung für die $x_{\mu}$ an; die zugehörigen $y_{\mu}$ entstehen daraus, indem man in den rechteckigen Teilen [die der rechten Hälfte des Schemas (III.3) entsprechen] die Vorzeichen umkehrt.

Die Einteilung der Matrizen (III.4) in 10-, 5- und 1-reihige Teile [sie ist auch im Schema (II.6) bereits angedeutet] ist von wesentlicher Bedeutung. Bildet man nämlich jetzt $\beta_{\mu}=x_{\mu}+y_{\mu}$, so hat man einfach die von 0 verschiedenen Elemente in den rechteckigen Teilen zu löschen und in den quadratischen zu verdoppeln. Die Darstellung der $\beta_{\mu}$, die man so erhalten hat, zerfällt also in eine 10-, 5- und 1-reihige Darstellung; in der letzteren werden die $\beta_{\mu}$ durch 0 dargestellt, aber $z_{\mu}$ durch - 1. Diese Darstellungen sind irreduzibel (und wir haben daher, wie oben gesagt,

$$
x_{3}=
$$

$\left(\begin{array}{rrrrrrrrrr|rrrrr|r}0 & 0 & 0 & 0 & 0 & 0 & 0 & 0 & 0 & 0 & 0 & 1 & 0 & 0 & 0 & 0 \\ 0 & 0 & 0 & 0 & 0 & 0 & 0 & 0 & 0 & 0 & -1 & 0 & 0 & 0 & 0 & 0 \\ 0 & 0 & 0 & 0 & 0 & 0 & 0 & 0 & 0 & 1 & 0 & 0 & 0 & 0 & 0 & 0 \\ 0 & 0 & 0 & 0 & 0 & 0 & 0 & -1 & 0 & 0 & 0 & 0 & 0 & 0 & 0 & 0 \\ 0 & 0 & 0 & 0 & 0 & 0 & 1 & 0 & 0 & 0 & 0 & 0 & 0 & 0 & 0 & 0 \\ 0 & 0 & 0 & 0 & 0 & 0 & 0 & 0 & 0 & 0 & 0 & 0 & 0 & -1 & 0 & 0 \\ 0 & 0 & 0 & 0 & 1 & 0 & 0 & 0 & 0 & 0 & 0 & 0 & 0 & 0 & 0 & 0 \\ 0 & 0 & 0 & -1 & 0 & 0 & 0 & 0 & 0 & 0 & 0 & 0 & 0 & 0 & 0 & 0 \\ 0 & 0 & 0 & 0 & 0 & 0 & 0 & 0 & 0 & 0 & 0 & 0 & 0 & 0 & 0 & 1 \\ 0 & 0 & 1 & 0 & 0 & 0 & 0 & 0 & 0 & 0 & 0 & 0 & 0 & 0 & 0 & 0 \\ \hline 0 & -1 & 0 & 0 & 0 & 0 & 0 & 0 & 0 & 0 & 0 & 0 & 0 & 0 & 0 & 0 \\ 1 & 0 & 0 & 0 & 0 & 0 & 0 & 0 & 0 & 0 & 0 & 0 & 0 & 0 & 0 & 0 \\ 0 & 0 & 0 & 0 & 0 & 0 & 0 & 0 & 0 & 0 & 0 & 0 & 0 & 0 & 1 & 0 \\ 0 & 0 & 0 & 0 & 0 & -1 & 0 & 0 & 0 & 0 & 0 & 0 & 0 & 0 & 0 & 0 \\ 0 & 0 & 0 & 0 & 0 & 0 & 0 & 0 & 0 & 0 & 0 & 0 & 1 & 0 & 0 & 0 \\ \hline 0 & 0 & 0 & 0 & 0 & 0 & 0 & 0 & 1 & 0 & 0 & 0 & 0 & 0 & 0 & 0\end{array}\right)$

$$
x_{4}=
$$

$\left(\begin{array}{rrrrrrrrrr|rrrrr|r}0 & 0 & 0 & 0 & 0 & 0 & -1 & 0 & 0 & 0 & 0 & 0 & 0 & 0 & 0 & 0 \\ 0 & 0 & 0 & 0 & 0 & 0 & 0 & -1 & 0 & 0 & 0 & 0 & 0 & 0 & 0 & 0 \\ 0 & 0 & 0 & 0 & 0 & 0 & 0 & 0 & -1 & 0 & 0 & 0 & 0 & 0 & 0 & 0 \\ 0 & 0 & 0 & 0 & 0 & 0 & 0 & 0 & 0 & 0 & 1 & 0 & 0 & 0 & 0 & 0 \\ 0 & 0 & 0 & 0 & 0 & 0 & 0 & 0 & 0 & 0 & 0 & 1 & 0 & 0 & 0 & 0 \\ 0 & 0 & 0 & 0 & 0 & 0 & 0 & 0 & 0 & 0 & 0 & 0 & 1 & 0 & 0 & 0 \\ -1 & 0 & 0 & 0 & 0 & 0 & 0 & 0 & 0 & 0 & 0 & 0 & 0 & 0 & 0 & 0 \\ 0 & -1 & 0 & 0 & 0 & 0 & 0 & 0 & 0 & 0 & 0 & 0 & 0 & 0 & 0 & 0 \\ 0 & 0 & -1 & 0 & 0 & 0 & 0 & 0 & 0 & 0 & 0 & 0 & 0 & 0 & 0 & 0 \\ 0 & 0 & 0 & 0 & 0 & 0 & 0 & 0 & 0 & 0 & 0 & 0 & 0 & 0 & 0 & 1 \\ \hline 0 & 0 & 0 & 1 & 0 & 0 & 0 & 0 & 0 & 0 & 0 & 0 & 0 & 0 & 0 & 0 \\ 0 & 0 & 0 & 0 & 1 & 0 & 0 & 0 & 0 & 0 & 0 & 0 & 0 & 0 & 0 & 0 \\ 0 & 0 & 0 & 0 & 0 & 1 & 0 & 0 & 0 & 0 & 0 & 0 & 0 & 0 & 0 & 0 \\ 0 & 0 & 0 & 0 & 0 & 0 & 0 & 0 & 0 & 0 & 0 & 0 & 0 & 0 & 1 & 0 \\ 0 & 0 & 0 & 0 & 0 & 0 & 0 & 0 & 0 & 0 & 0 & 0 & 0 & 1 & 0 & 0 \\ \hline 0 & 0 & 0 & 0 & 0 & 0 & 0 & 0 & 0 & 1 & 0 & 0 & 0 & 0 & 0 & 0\end{array}\right)$


alle irreduziblen Darstellungen der $\beta$-Algebra gefunden). Denn da man in jeder irreduziblen Darstellung noch die in ihr enthaltene Darstellung der $z$-Algebra ausreduzieren kann, kann jede irreduzible Darstellung als Teil einer der Darstellungen geschrieben werden, die man auf die soeben geschilderte Art erhalten kann, und dabei ist durch die Reihenfolge der Spalten des Schemas (II.6) schon festgelegt, an welchen Stellen die 8 von Null verschiedenen Matrixelemente stehen. Man überzeugt sich leicht, daß diese Reihenfolge bereits möglichst günstig gewählt ist, so daß man durch ihre Abänderung nicht weiter reduzieren kann.

Es folgt, daß die Dimension der $\beta$-Algebra $10^{2}+5^{2}+1^{2}=126$ ist. Übrigens ist es nicht schwer, mit Hilfe der Vertauschungsrelationen (I.6) eine Basis aus $126 \beta$ - und $z$-Produkten wirklich aufzustellen ${ }^{15}$.

\section{Der F all $N=3$}

Wir betrachten jetzt die aus drei vertauschbaren $\alpha$-Systemen $x_{\mu}, y_{\mu}, z_{\mu}$ gebildete „ $x$ - $y$ - $z$-Algebra “ und behandeln die Aufgabe, die irreduziblen Darstellungen der in ihr enthaltenen, durch die Elemente $\gamma_{\mu}=x_{\mu}+y_{\mu}+z_{\mu}$ erzeugten $\gamma$-Algebra zu finden, die wir mit den $\gamma_{\mu}^{(3)}$ von (I.4) identifizieren wollen. Wir setzen dazu noch $u_{\mu}=y_{\mu} z_{\mu}, v_{\mu}=x_{\mu} z_{\mu}$, $w_{\mu}=x_{\mu} y_{\mu}$. Jedes dieser drei Systeme ist ein $\eta$-System; man verifiziert leicht (II.4). Außerdem ist

$$
\begin{gathered}
u_{\mu} v_{\mu}=v_{\mu} u_{\mu}=w_{\mu}, \quad u_{\mu} w_{\mu}=w_{\mu} u_{\mu}=v_{\mu} \\
v_{\mu} w_{\mu}=w_{\mu} v_{\mu}=u_{\mu} \\
\begin{aligned}
u_{\mu} v_{\nu}+v_{\nu} u_{\mu}= & u_{\mu} w_{\nu}+w_{\nu} u_{\mu} \\
= & v_{\mu} w_{\nu}+w_{\nu} v_{\mu}=0 \quad(\mu \neq \nu) .
\end{aligned}
\end{gathered}
$$

Endlich ist $\zeta_{\mu}=x_{\mu} y_{\mu} z_{\mu}$ ein $\alpha$-System; für diese Größen gilt u. a.

$$
\begin{gathered}
\zeta_{\mu} u_{\mu}=u_{\mu} \zeta_{\mu}=x_{\mu}, \quad \zeta_{\mu} v_{\mu}=v_{\mu} \zeta_{\mu}=y_{\mu}, \\
\zeta_{\mu} w_{\mu}=w_{\mu} \zeta_{\mu}=z_{\mu} ; \\
\zeta_{\mu} u_{\nu}-u_{\nu} \zeta_{\mu}=\zeta_{\mu} v_{\nu}-v_{\nu} \zeta_{\mu} \\
=\zeta_{\mu} w_{\nu}-w_{\nu} \zeta_{\mu}=0 .
\end{gathered}
$$

Die $\zeta$-Algebra liegt in der $\gamma$-Algebra:

$$
\zeta_{\mu}=\frac{1}{6}\left(\gamma_{\mu}^{3}-7 \gamma_{\mu}\right)
$$

Für die $x$-y-z-Algebra ist

$x_{\mu}=c_{\mu} \times 1 \times 1, y_{\mu}=1 \times a_{\mu} \times 1, z_{\mu}=1 \times 1 \times c_{\mu}$ eine 64-reihige treue Darstellung; wir folgern wieder, daß sie die einzige irreduzible Darstellung ist und alle irreduziblen Darstellungen der $\gamma$ - und der $\zeta$-Algebra enthält, weil auch diese treu dargestellt werden. Merken wir noch an, weil wir es gleich benutzen werden, daß $\mathrm{u}$. a. auch das $\eta$-System $w_{\mu}$ treu dargestellt wird (nämlich durch $\alpha_{\mu} \times \alpha_{\mu} \times 1$ ).

Man reduziert wieder zuerst das $\alpha$-System $\zeta_{\mu}$ aus; hier mag sich 16-mal die eine Darstellung $\alpha_{\mu}$ wiederholen:

$$
\zeta_{\mu} \sim 1 \times \alpha_{\mu} .
$$

Dabei bezeichnet 1 die 16-reihige Einsmatrix, und die Reihenfolge der Zeilen und Spalten des KroneckerProdukts ist hier wie im folgenden so gedacht, daß es sich um 16-reihige Matrizen aus 4-reihigen Kästchen handelt.

Da nur die Multipla der Einsmatrix mit allen $\alpha_{\mu}$ vertauschbar sind, fordert die Vertauschbarkeit (IV.2)

$$
u_{\mu} \sim U_{\mu} \times 1, \quad v_{\mu} \sim V_{\mu} \times 1, w_{\mu} \sim W_{\mu} \times 1
$$

mit 16-reihigen Matrizen $U, V, W$, die ebenfalls alle Relationen (IV.1) erfüllen, und 4-reihigen Einsmatrizen; und man findet weiter

$$
\begin{gathered}
x_{\mu}=u_{\mu} \xi_{\mu} \sim U_{\mu} \times c_{\mu}, \quad y_{\mu} \sim V_{\mu} \times c_{\mu}, \\
z_{\mu} \sim W_{\mu} \times c_{\mu} .
\end{gathered}
$$

Da man in jeder Darstellung die $\zeta_{\mu}$ ausreduziert annehmen, sie also wie(IV. 3) mit zweitem KroneckerFaktor $\alpha_{\mu}$ schreiben kann, können wir die weitere Reduktion durch Transformation mittels Matrizen $C \times 1$, mit 16-reihigem $C$, bewerkstelligen: d. h. wir brauchen uns nur noch um die 16-reihigen Matrizen $U, V, W$ zu kümmern.

Reduzieren wir zuerst die $W_{\mu}$, als 16-reihige treue Darstellung der $\eta$-Algebra, wie im vorigen Abschnitt aus. Die Relationen (IV.1) zwischen $u_{\mu}, v_{\mu}$ und $w_{\mu}$ unterscheiden sich von den Relationen (III. 1), (III. 2) für die dortigen $x_{\mu}, y_{\mu}$ und $z_{\mu}$ in einigenVorzeichen. Man überzeugt sich, daß man in analoger Weise wie dort (IV.1) erfüllen kann und daß man diesmal eine Lösung erhält, wenn man für $U_{\mu}$ dieselben Matrizen wie (III.4), aber nur mit + -Zeichen nimmt. $V_{\mu}$ hat dann in den rechteckigen Teilen lauter $-1 . U_{\mu}+V_{\mu}$ zerfällt in eine 10-, 5- und 1-reihige Matrix, und das-

15 Die von uns hingeschriebenen Darstellungen sind etwas einfacher als die von Kemmer angegebenen, insbesondere sind sie durchweg reell. In der 10-reihigen Darstellung stehen die von 0 verschiedenen Elemente an denselben Stellen wie bei Kemmer, in der 5-reihigen entsprechen Kemmers Matrizen einer anderen Wahl der Reihenfolge der Spalten 11 bis 14 von (II.6). 
selbe gilt für die Summe

$$
\Gamma_{\mu}=U_{\mu}+V_{\mu}+W_{\mu},
$$

deren Bestandteile wir mit $\Gamma_{\mu}^{(1)}=-1, \Gamma_{\mu}^{(5)}, \Gamma_{\mu}^{(10)}$ bezeichnen.

Aus (IV.1) folgt noch

$$
\begin{gathered}
I_{\mu}^{2}=2 \Gamma_{\mu}+3 \text { oder } \\
\left(\Gamma_{\mu}-3\right)\left(\Gamma_{\mu}+1\right)=0 .
\end{gathered}
$$

Für $\gamma_{\mu}$ haben wir dann

$$
\gamma_{\mu} \sim \Gamma_{\mu} \times \iota_{\mu}
$$

also drei Darstellungen der Grade 10·4, 5.4, 1.4. Sie sind nicht irreduzibel; im folgenden schreiben wir die Matrizen $\Gamma_{\mu}$ der Reihe nach hin und reduzieren sie zu Ende.

V. Die vollständige Reduktion der $\gamma_{\mu}$ a) $\Gamma_{\mu}^{(1)}=-1$ ist natürlich schon irreduzibel. Eigenwert -1 , Eigenwerte der $\gamma_{\mu} \sim-\iota_{\mu}: \pm 1$.

$$
\begin{aligned}
& \Gamma_{1}{ }^{(5)}=\left(\begin{array}{rrrrr}
1 & 0 & 0 & 0 & 2 \\
0 & -1 & 0 & 0 & 0 \\
0 & 0 & -1 & 0 & 0 \\
0 & 0 & 0 & -1 & 0 \\
2 & 0 & 0 & 0 & 1
\end{array}\right), \Gamma_{2}{ }^{(5)}=\left(\begin{array}{rrrrr}
-1 & 0 & 0 & 0 & 0 \\
0 & 1 & 0 & 0 & 2 \\
0 & 0 & -1 & 0 & 0 \\
0 & 0 & 0 & -1 & 0 \\
0 & 2 & 0 & 0 & 1
\end{array}\right) \text {, } \\
& \Gamma_{3}{ }^{(5)}=\left(\begin{array}{rrrrr}
-1 & 0 & 0 & 0 & 0 \\
0 & -1 & 0 & 0 & 0 \\
0 & 0 & 1 & 0 & 2 \\
0 & 0 & 0 & -1 & 0 \\
0 & 0 & 2 & 0 & 1
\end{array}\right), \quad I_{4}{ }^{(5)}=\left(\begin{array}{rrrrr}
-1 & 0 & 0 & 0 & 0 \\
0 & -1 & 0 & 0 & 0 \\
0 & 0 & -1 & 0 & 0 \\
0 & 0 & 0 & 1 & 2 \\
0 & 0 & 0 & 2 & 1
\end{array}\right) \text {. }
\end{aligned}
$$

Eigenwerte: $3,-1,-1,-1,-1$. Es ist sehr leicht, zugehörige Eigenvektoren hinzuschreiben. $\mathrm{Zu}$ -1 gibt es den gemeinsamen Eigenvektor $(1,1,1,1$, -1), also spaltet sich noch eine Darstellung $\Gamma_{u}^{(1)}$ ab. Was übrig bleibt, muß irreduzibel sein. Denn da die „Austauschbarkeit“ der $\alpha_{\mu}$ (vgl. S. 355) die der $\gamma_{\mu}$ bedingt, müssen in jeder Darstellung für alle Indizes $\mu$ die gleichen Eigenwerte vorkommen und daher die vier Eigenvektoren zum Eigenwert 3,

$$
\begin{aligned}
& (1,0,0,0,1) \\
& (0,1,0,0,1) \\
& (0,0,1,0,1) \\
& (0,0,0,1,1)
\end{aligned}
$$

im gleichen invarianten Teilraum liegen; da sie linear unabhängig sind, spannen sie ihn auf. Nimmt man sie als Basisvektoren, so lautet die so gewonnene $4 \cdot 4$ reihige irreduzible Darstellung:

$$
\begin{gathered}
\Gamma_{1}^{(4)}=\left(\begin{array}{rrrr}
3 & 2 & 2 & 2 \\
0 & -1 & 0 & 0 \\
0 & 0 & -1 & 0 \\
0 & 0 & 0 & -1
\end{array}\right), \Gamma_{2}^{(4)}=\left(\begin{array}{rrrr}
-1 & 0 & 0 & 0 \\
2 & 3 & 2 & 2 \\
0 & 0 & -1 & 0 \\
0 & 0 & 0 & -1
\end{array}\right), \\
\Gamma_{3}^{(4)}=\left(\begin{array}{rrrr}
-1 & 0 & 0 & 0 \\
0 & -1 & 0 & 0 \\
2 & 2 & 3 & 2 \\
0 & 0 & 0-1
\end{array}\right), I_{4}^{(4)}=\left(\begin{array}{rrrr}
-1 & 0 & 0 & 0 \\
0 & -1 & 0 & 0 \\
0 & 0 & -1 & 0 \\
2 & 2 & 2 & 3
\end{array}\right) ; \\
\gamma_{\mu} \sim I_{\mu}^{(4)} \times \iota_{\mu} .
\end{gathered}
$$

Sie ist nicht hermitesch; würde man, von (V.1) ausgehend, unitär transformieren, um die hermitesche Form zu erhalten, so würden Quadratwurzeln auftreten. Eigenwerte der $\gamma_{\mu}: \pm 3$ (je 2 -fach), \pm 1 (je 6-fach).

$$
\begin{aligned}
& \text { c) } \Gamma_{1}{ }^{(10)}=\left(\begin{array}{rrrrrrrrrr}
1 & 0 & 0 & 0 & 0 & 0 & 0 & 0 & 0 & 2 \\
0 & -1 & 0 & 0 & 0 & 0 & 0 & 0 & 0 & 0 \\
0 & 0 & -1 & 0 & 0 & 0 & 0 & 0 & 0 & 0 \\
0 & 0 & 0 & -1 & 0 & 0 & 0 & 0 & 0 & 0 \\
0 & 0 & 0 & 0 & 1 & 0 & 0 & 0 & 2 & 0 \\
0 & 0 & 0 & 0 & 0 & 1 & 0 & 2 & 0 & 0 \\
0 & 0 & 0 & 0 & 0 & 0 & -1 & 0 & 0 & 0 \\
0 & 0 & 0 & 0 & 0 & 2 & 0 & 1 & 0 & 0 \\
0 & 0 & 0 & 0 & 2 & 0 & 0 & 0 & 1 & 0 \\
2 & 0 & 0 & 0 & 0 & 0 & 0 & 0 & 0 & 1
\end{array}\right) \text {, } \\
& \Gamma_{2}{ }^{(10)}=\left(\begin{array}{rrrrrrrrrr}
-1 & 0 & 0 & 0 & 0 & 0 & 0 & 0 & 0 & 0 \\
0 & 1 & 0 & 0 & 0 & 0 & 0 & 0 & 0 & 2 \\
0 & 0 & -1 & 0 & 0 & 0 & 0 & 0 & 0 & 0 \\
0 & 0 & 0 & 1 & 0 & 0 & 0 & 0 & 2 & 0 \\
0 & 0 & 0 & 0 & -1 & 0 & 0 & 0 & 0 & 0 \\
0 & 0 & 0 & 0 & 0 & 1 & 2 & 0 & 0 & 0 \\
0 & 0 & 0 & 0 & 0 & 2 & 1 & 0 & 0 & 0 \\
0 & 0 & 0 & 0 & 0 & 0 & 0 & -1 & 0 & 0 \\
0 & 0 & 0 & 2 & 0 & 0 & 0 & 0 & 1 & 0 \\
1 & 2 & 0 & 0 & 0 & 0 & 0 & 0 & 0 & 1
\end{array}\right) \text {, }
\end{aligned}
$$

$$
\Gamma_{3}{ }^{(10)}=\left(\begin{array}{rrrrrrrrrr}
-1 & 0 & 0 & 0 & 0 & 0 & 0 & 0 & 0 & 0 \\
0 & -1 & 0 & 0 & 0 & 0 & 0 & 0 & 0 & 0 \\
0 & 0 & 1 & 0 & 0 & 0 & 0 & 0 & 0 & 2 \\
0 & 0 & 0 & 1 & 0 & 0 & 0 & 2 & 0 & 0 \\
0 & 0 & 0 & 0 & 1 & 0 & 2 & 0 & 0 & 0 \\
0 & 0 & 0 & 0 & 0 & -1 & 0 & 0 & 0 & 0 \\
0 & 0 & 0 & 0 & 2 & 0 & 1 & 0 & 0 & 0 \\
0 & 0 & 0 & 2 & 0 & 0 & 0 & 1 & 0 & 0 \\
0 & 0 & 0 & 0 & 0 & 0 & 0 & 0 & -1 & 0 \\
0 & 0 & 2 & 0 & 0 & 0 & 0 & 0 & 0 & 1
\end{array}\right),
$$

$$
\Gamma_{4}^{(10)}=\left(\begin{array}{rrrrrrrrrr}
1 & 0 & 0 & 0 & 0 & 0 & 2 & 0 & 0 & 0 \\
0 & 1 & 0 & 0 & 0 & 0 & 0 & 2 & 0 & 0 \\
0 & 0 & 1 & 0 & 0 & 0 & 0 & 0 & 2 & 0 \\
0 & 0 & 0 & -1 & 0 & 0 & 0 & 0 & 0 & 0 \\
0 & 0 & 0 & 0 & -1 & 0 & 0 & 0 & 0 & 0 \\
0 & 0 & 0 & 0 & 0 & -1 & 0 & 0 & 0 & 0 \\
2 & 0 & 0 & 0 & 0 & 0 & 1 & 0 & 0 & 0 \\
0 & 2 & 0 & 0 & 0 & 0 & 0 & 1 & 0 & 0 \\
0 & 0 & 2 & 0 & 0 & 0 & 0 & 0 & 1 & 0 \\
0 & 0 & 0 & 0 & 0 & 0 & 0 & 0 & 0 & -1
\end{array}\right) .
$$


Eigenwerte: je 3-mal 3 und 7-mal - 1. Linear unabhängige Eigenvektoren jeder Matrix zum Eigenwert 3 sind $\mathrm{z}$. B.:

$$
\begin{array}{cc}
\Gamma_{1}: & \Gamma_{2}: \\
x_{1}=e_{1}+e_{10}, & y_{1}=e_{2}+e_{10}, \\
x_{2}=e_{5}+e_{9}, & y_{2}=e_{4}+e_{9}, \\
x_{3}=e_{6}+e_{8}, & y_{3}=e_{6}+e_{7}, \\
& \Gamma_{3}: \\
z_{1}=e_{3}+e_{10}, & { }_{4}: \\
z_{2}=e_{4}+e_{8}, & w_{2}=e_{2}+e_{8}, \\
z_{3}=e_{5}+e_{7}, & w_{3}=e_{3}+e_{9},
\end{array}
$$

wenn $e_{j}=\left(\delta_{j k}\right)$ der $j$-te Koordinatenvektor ist. Wieder gibt es einen gemeinsamen Eigenvektor zum Eigenwert - 1, nämlich $(1,1,1,1,1,1,-1,-1$, $-1,-1)$, also ein drittes Mal die Darstellung $\Gamma_{u}^{(1)}$. Weiter sind die Vektoren

$$
\begin{array}{ll}
x=x_{1}+x_{2}+x_{3}, & y=y_{1}+y_{2}+y_{3}, \\
z=z_{1}+z_{2}+z_{3}, & w=w_{1}+w_{2}+w_{3}
\end{array}
$$

linear unabhängig und transformieren sich untereinander nach der Darstellung $\Gamma_{\mu}^{(4)}$, die also zum zweitenmal auftritt. Was übrig bleibt, ist irreduzibel. In der Tat, da es keinen gemeinsamen Eigenvektor zum Eigenwert 3 und keinen weiteren gemeinsamen zu -1 gibt, käme nur die Zerlegung $5=3+2$ in Frage, wobei in jedem Teilraum für jedes $\mu$ ein Eigenvektor zu 3 vorkommen müßte. Nun ist für jeden Vektor $u$, wie aus (IV.4) folgt, $\Gamma_{u} u+u$ Eigenvektor von $\Gamma_{\mu}$ zum Eigenwert 3; insbesondere werden durch die Operationen $\Gamma_{\mu}+1$ die Eigenvektoren zu 3 stets untereinander transformiert. Würde man z. B. in einem der beiden Teilräume auf den Eigenvektor $u$ von $\Gamma_{1}$, also eine zu dem obigen $x$ senkrechte Linearkombination von $x_{1}, x_{2}, x_{3}$, die Operation $\Gamma_{3}+1$ oder zuerst $\Gamma_{2}+1$ und dann $\Gamma_{3}+1$ ausüben, man müßte jedesmal zu dem in diesem Teilraum liegenden Eigenvektor von $I_{3}$ geführt werden, d. h. $\Gamma_{3} u+u$ und $\Gamma_{3}\left(\Gamma_{2} u+u\right)+\left(\Gamma_{2} u+u\right)$ wären proportional. Man rechnet leicht nach, daß es eine solche Linearkombination von $x_{1}, x_{2}, x_{3}$ nicht gibt.

Als dritte irreduzible Darstellung ergibt sich so eine vom Grad 5•4. Nimmt man als Koordinatensystem (eine Auswahl aus den Eigenvektoren zu treffen ist nur in sehr asymmetrischer Weise möglich) z. B. die Vektoren $x_{1}-x_{2}, x_{2}-x_{3}, y_{1}-y_{2}, y_{2}-y_{3}$, $z_{1}-z_{2}$, so erhält man die Matrizen

$\hat{\Gamma}_{1}{ }^{(5)}=\left(\begin{array}{rrrrr}3 & 0 & 2 & 0 & 2 \\ 0 & 3 & 0 & 2 & 2 \\ 0 & 0 & -1 & 0 & 0 \\ 0 & 0 & 0 & -1 & 0 \\ 0 & 0 & 0 & 0 & -1\end{array}\right), \hat{\Gamma}_{2}{ }^{(5)}=\left(\begin{array}{rrrrr}-1 & 0 & 0 & 0 & 0 \\ 0 & -1 & 0 & 0 & 0 \\ 2 & 0 & 3 & 0 & 2 \\ 0 & 2 & 0 & 3 & 0 \\ 0 & 0 & 0 & 0 & -1\end{array}\right)$

$$
\begin{aligned}
\hat{\Gamma}_{3}{ }^{(5)}= & \left(\begin{array}{rrrrr}
-1 & 0 & 0 & 0 & 0 \\
-2 & 1 & 0 & -2 & 0 \\
0 & 0 & -1 & 0 & 0 \\
2 & -2 & 0 & 1 & 0 \\
2 & 0 & 2 & 0 & 3
\end{array}\right), \hat{\Gamma}_{4}^{(5)}=\left(\begin{array}{rrrrr}
1 & 0 & 0-2 & 0 \\
2-1 & 0-2 & 0 \\
0-2 & 1 & 0-2 \\
-2 & 0 & 0 & 1 & 0 \\
-2 & 2-2 & 2 & 1
\end{array}\right) ; \\
\gamma_{\mu} \sim \hat{\Gamma}_{\mu}^{(5)} \times \iota_{\mu} . &
\end{aligned}
$$

Eigenwerte von $\gamma_{\mu}$ : \pm 3 (je 4 -fach), \pm 1 (je 6-fach). Die untersuchte Algebra der $\gamma_{\mu}=\gamma_{\mu}^{(3)}$ ist also von der Dimension $4^{2}+16^{2}+20^{2}=672$ (vgl. ${ }^{14}$ ).

Für die Spinkomponenten (I.5) erhält man durch Einsetzen von (IV.5) (in Einheiten von $\hbar$ )

$$
\begin{aligned}
S_{i k}= & \frac{1}{4 i}\left(\Gamma_{j} \Gamma_{k}+\Gamma_{k} \Gamma_{j}\right) \times\left(a_{j} a_{k}\right) \\
& ((j, k)=(1,2), \quad(2,3), \quad(3,1)) ;
\end{aligned}
$$

ihre Quadratsumme, der „Gesamtspin“, ist

$$
S^{2}=\frac{1}{16} \sum_{i, k}\left(\Gamma_{i} \Gamma_{k}+\Gamma_{k} \Gamma_{j}\right)^{2} \times 1 .
$$

$S^{2}$ hat die Eigenwerte $3 / 4$ und $15 / 4$.

\section{Die Wellengleichungen für $N=3$}

Um die Wellengleichung

$$
\left(\gamma_{\mu} \partial_{\mu}+3 \varkappa\right) \psi=0
$$

in ausreduzierter Form (d. h. die Wellengleichungen, die den verschiedenen irreduziblen Darstellungen entsprechen) angeben zu können, ist es nicht immer zweckmäßig, die berechneten Matrizen wirklich zu benutzen. Es ist vielfach praktischer, von einer geeigneten nicht ganz reduzierten Form auszugehen und die Reduktion in einfacher Weise durch Hinzufügung von geeigneten Nebenbedingungen $\mathrm{zu}$ bewirken (Methode von F. L. B a u e $\mathrm{r}^{5}$ ).

a) Die Wellengleichungen der (4.4)-reihigen Darstellung (V.2)

Wir gehen von den Definitionen

$$
\begin{gathered}
\beta_{\mu}=a_{\mu} \times 1+1 \times a_{\mu}, \\
\gamma_{\mu}=c_{\mu} \times 1 \times 1+1 \times c_{\mu} \times 1+1 \times 1 \times a_{\mu} \\
=\beta_{\mu} \times 1+1 \times c_{\mu}
\end{gathered}
$$

aus, setzen hier für $\beta_{\mu}$ die ausreduzierte Form an (die quadratischen Kästchen aus (III.4), mit 2 multipliziert) und beschränken uns zuerst auf die 5-reihigen Kästchen. Wenn wir das Kronecker-Produkt wie in IV anordnen, können wir also 
$\gamma_{1}=\left(\begin{array}{ccccc}c_{1} & 0 & 0 & 0 & 2 \\ 0 & c_{1} & 0 & 0 & 0 \\ 0 & 0 & c_{1} & 0 & 0 \\ 0 & 0 & 0 & a_{1} & 0 \\ 2 & 0 & 0 & 0 & a_{1}\end{array}\right), \quad \gamma_{2}=\left(\begin{array}{ccccc}c_{2} & 0 & 0 & 0 & 0 \\ 0 & c_{2} & 0 & 0 & 2 \\ 0 & 0 & c_{2} & 0 & 0 \\ 0 & 0 & 0 & c_{2} & 0 \\ 0 & 2 & 0 & 0 & c_{2}\end{array}\right)$,

(VI. 3)

$$
\gamma_{3}=\left(\begin{array}{ccccc}
a_{3} & 0 & 0 & 0 & 0 \\
0 & c_{3} & 0 & 0 & 0 \\
0 & 0 & c_{3} & 0 & 2 \\
0 & 0 & 0 & c_{3} & 0 \\
0 & 0 & 2 & 0 & c_{3}
\end{array}\right), \quad \gamma_{4}=\left(\begin{array}{ccccc}
c_{4} & 0 & 0 & 0 & 0 \\
0 & c_{4} & 0 & 0 & 0 \\
0 & 0 & c_{4} & 0 & 0 \\
0 & 0 & 0 & c_{4} & 2 \\
0 & 0 & 0 & 2 & c_{4}
\end{array}\right)
$$

nehmen; hier bedeutet jedes Element ein 4-reihiges quadratisches Kästchen, also z. B. „2“ die doppelte 4-reihige Einsmatrix.

Die $\psi$-Funktion muß aus 5 Komponenten $\psi_{1}, \ldots, \psi_{5}$ bestehen, deren jede wieder ein Vektor aus $4 \mathrm{Kom}$ ponenten ist, die wir nicht erst zu kennzeichnen brauchen. Jetzt ist es leicht, die Gl. (VI. 1) hinzuschreiben; es sind 5 Gleichungen, in denen nur noch 4-reihige Matrizen und Vektoren vorkommen:

$$
\begin{gathered}
\left(a_{\mu} \partial_{\mu}+3 \varkappa\right) \psi_{\lambda}+2 \partial_{\lambda} \psi_{5}=0 ; \quad(\lambda=1, \ldots, 4) \\
2 \partial_{\mu} \psi_{\mu}+\left(a_{\mu} \partial_{\mu}+3 \varkappa\right) \psi_{5}=0 .
\end{gathered}
$$

Transformiert man $\psi$ durch eine Matrix $T: \psi^{\prime}=T \psi$, so hat man $\gamma_{\mu}$ durch $\gamma_{\mu}^{\prime}=T \gamma_{\mu} T^{-1}$ zu ersetzen. Wir wählen

$$
T=\left(\begin{array}{ccccc}
a_{1} & 0 & 0 & 0 & 0 \\
0 & a_{2} & 0 & 0 & 0 \\
0 & 0 & a_{3} & 0 & 0 \\
0 & 0 & 0 & a_{4} & 0 \\
0 & 0 & 0 & 0 & 1
\end{array}\right)
$$

dann ist $T^{-1}=T$ und $\psi_{\mu}^{\prime}=\alpha_{\mu} \psi_{\mu}, \psi_{5}^{\prime}=\psi_{5}$, und die Matrizen (VI.3) gehen über in

$$
\gamma_{\mu^{\prime}}{ }^{\prime}=\Gamma_{\mu}{ }^{(5)} \times \iota_{\mu}
$$

mit den Matrizen (V.1), die wir dort auf anderem Wege fanden. Um uns auf die Darstellung (V.2) zu beschränken, betrachten wir zuerst den Teilraum, der zu der abzuspaltenden (1.4)-reihigen Darstellung gehört. Er gehört zum gemeinsamen Eigenvektor $(1,1$,

16 Dies folgt aus dem Satz, daß bei Hermiteschen Matrixsystemen mit jedem invarianten Teilraum auch der dazu total senkrechte Teilraum invariant ist, zusammen mit der Eindeutigkeit der Zerlegung in irreduzible Bestandteile.

17 Man multipliziere (VI. 6) mit $\alpha_{\lambda}$ und summiere über $\lambda$. Dann kommt

$$
\begin{aligned}
0 & =\left(a_{\lambda} a_{\mu}+2 a_{\mu} a_{\lambda}\right) \partial_{\mu} \psi_{\lambda}+3 \% a_{\lambda} \psi_{\lambda} \\
& =2 \delta_{\lambda \mu} \partial_{\mu} \psi_{\lambda}+\left(a_{\mu} \partial_{\mu}+3 \varkappa\right) a_{\lambda} \psi_{\lambda} \\
& =2 \partial_{\mu} \psi_{\mu}+\left(a_{\mu} \partial_{\mu}+3 \alpha a_{\lambda} \psi_{\lambda} .\right.
\end{aligned}
$$

$1,1,-1) \operatorname{der} \Gamma_{\mu}^{(5)}$, besteht also aus allen Vektoren $\psi^{\prime}$, bei denen $\psi_{1}^{\prime}=\psi_{2}^{\prime}=\psi_{3}^{\prime}=\psi_{4}^{\prime}=-\psi_{5}^{\prime}$ ist. Der gesuchte $(4 \cdot 4)$-reihige besteht aus allen Vektoren, die auf allen diesen senkrecht stehen ${ }^{16}$, ist also durch

$$
\psi_{1}{ }^{\prime}+\psi_{2}{ }^{\prime}+\psi_{3}{ }^{\prime}+\psi_{4}{ }^{\prime}-\psi_{5}{ }^{\prime}=0
$$

zu kennzeichnen. Das ist die gesuchte Nebenbedingung, wir müssen nur noch $\psi^{\prime}$ durch $\psi$ ausdrücken und erhalten

$$
\psi_{5}=c_{u l} \psi_{i l} \text {. }
$$

(VI.4) und (VI.5) bilden das gesuchte System der Wellengleichungen zur Darstellung (V.2). Man kann noch $\psi_{5}$ eliminieren:

$\left(c_{\mu} \partial_{\mu}+3 \%\right) \psi_{\lambda}+2 \partial_{\lambda} c_{\mu} \psi_{\mu}=0 \quad(\lambda=1, \ldots, 4) ;$

die fünfte Gleichung ist jetzt eine Folge von diesen ${ }^{17}$.

Wenn man zu (VI.6) die Matrizen aufschreibt und mit

$$
\left(\begin{array}{cccc}
c_{1} & 0 & 0 & 0 \\
0 & c_{2} & 0 & 0 \\
0 & 0 & c_{3} & 0 \\
0 & 0 & 0 & c_{4}
\end{array}\right)
$$

transformiert, so erhält man übrigens genau (V.2); es erschien aber nicht überflüssig, auch schon (VI.6) auf dem Weg herzuleiten, der uns bei der anderen Darstellung dienen wird.

b) Die Wellengleichungen der (5.4)-reihigen Darstellung (V.4)

Wir benutzen wieder (VI. 2) und setzen für $\beta_{\mu}$ die 10-reihigen Kästchen aus (III. 4) ein:

$$
\gamma_{1}=\left(\begin{array}{cccccccccc}
c_{1} & 0 & 0 & 0 & 0 & 0 & 0 & 0 & 0 & 2 \\
0 & a_{1} & 0 & 0 & 0 & 0 & 0 & 0 & 0 & 0 \\
0 & 0 & c_{1} & 0 & 0 & 0 & 0 & 0 & 0 & 0 \\
0 & 0 & 0 & c_{1} & 0 & 0 & 0 & 0 & 0 & 0 \\
0 & 0 & 0 & 0 & c_{1} & 0 & 0 & 0 & -2 & 0 \\
0 & 0 & 0 & 0 & 0 & c_{1} & 0 & 2 & 0 & 0 \\
0 & 0 & 0 & 0 & 0 & 0 & c_{1} & 0 & 0 & 0 \\
0 & 0 & 0 & 0 & 0 & 2 & 0 & c_{1} & 0 & 0 \\
0 & 0 & 0 & 0 & -2 & 0 & 0 & 0 & c_{1} & 0 \\
2 & 0 & 0 & 0 & 0 & 0 & 0 & 0 & 0 & c_{1}
\end{array}\right),
$$




$$
\begin{aligned}
& \gamma_{3}=\left(\begin{array}{cccccccccc}
c_{3} & 0 & 0 & 0 & 0 & 0 & 0 & 0 & 0 & 0 \\
0 & c_{3} & 0 & 0 & 0 & 0 & 0 & 0 & 0 & 0 \\
0 & 0 & c_{3} & 0 & 0 & 0 & 0 & 0 & 0 & 2 \\
0 & 0 & 0 & c_{3} & 0 & 0 & 0 & -2 & 0 & 0 \\
0 & 0 & 0 & 0 & c_{3} & 0 & 2 & 0 & 0 & 0 \\
0 & 0 & 0 & 0 & 0 & c_{3} & 0 & 0 & 0 & 0 \\
0 & 0 & 0 & 0 & 2 & 0 & c_{3} & 0 & 0 & 0 \\
0 & 0 & 0 & -2 & 0 & 0 & 0 & c_{3} & 0 & 0 \\
0 & 0 & 0 & 0 & 0 & 0 & 0 & 0 & c_{3} & 0 \\
0 & 0 & 2 & 0 & 0 & 0 & 0 & 0 & 0 & c_{3}
\end{array}\right) \\
& \gamma_{4}=\left(\begin{array}{rrrrrrrrrr}
c_{4} & 0 & 0 & 0 & 0 & 0 & -2 & 0 & 0 & 0 \\
0 & c_{4} & 0 & 0 & 0 & 0 & 0 & -2 & 0 & 0 \\
0 & 0 & c_{4} & 0 & 0 & 0 & 0 & 0 & -2 & 0 \\
0 & 0 & 0 & c_{4} & 0 & 0 & 0 & 0 & 0 & 0 \\
0 & 0 & 0 & 0 & c_{4} & 0 & 0 & 0 & 0 & 0 \\
0 & 0 & 0 & 0 & 0 & c_{4} & 0 & 0 & 0 & 0 \\
-2 & 0 & 0 & 0 & 0 & 0 & c_{4} & 0 & 0 & 0 \\
0 & -2 & 0 & 0 & 0 & 0 & 0 & c_{4} & 0 & 0 \\
0 & 0 & -2 & 0 & 0 & 0 & 0 & 0 & a_{4} & 0 \\
0 & 0 & 0 & 0 & 0 & 0 & 0 & 0 & 0 & c_{4}
\end{array}\right) .
\end{aligned}
$$$$
T=\left(\begin{array}{ccccc}
c_{2} c_{3} & 0 & 0 & 0 & 0 \\
0 & c_{3} c_{1} & 0 & 0 & 0 \\
0 & 0 & c_{1} c_{2} & 0 & 0 \\
0 & 0 & 0 & c_{1} c_{4} & 0 \\
0 & 0 & 0 & 0 & c_{2} c_{4} \\
0 & 0 & 0 & 0 & 0 \\
0 & 0 & 0 & 0 & 0 \\
0 & 0 & 0 & 0 & 0 \\
0 & 0 & 0 & 0 & 0 \\
0 & 0 & 0 & 0 & 0
\end{array}\right.
$$

Es ist also $\psi_{14}^{\prime}=\alpha_{2} \alpha_{3} \psi_{14}$ usw., $T^{-1}=-T^{18}$, und aus $\gamma_{\mu}$ wird gerade

$$
\gamma_{\mu}^{\prime}=\Gamma_{\mu}^{(10)} \times a_{\mu}
$$

mit den Matrizen (V.3). (Die eigentümliche Verschiedenheit der Vorzeichen in III und IV erfährt hier eine neue Beleuchtung.)

Unsere Aufgabe ist, durch Nebenbedingungen aus der Zerlegung $5+4+1$ dieser Matrizen die 4- und die 1-reihige Darstellung auszuscheiden. Nach V wird der eindimensionale invariante Teilraum der $\Gamma_{\mu}^{(10)}$ durch den Vektor $(1,1,1,1,1,1,-1,-1,-1,-1)$, der 4-dimensionale durch die Vektoren

$$
\begin{aligned}
x & =(1,0,0,0,1,1,0,1,1,1), \\
y & =(0,1,0,1,0,1,1,0,1,1), \\
z & =(0,0,1,1,1,0,1,1,0,1), \\
w & =(1,1,1,0,0,0,1,1,1,0)
\end{aligned}
$$

18 Die Dreierprodukte sind bis auf eine gerade Permutation der Faktoren festgelegt; sie sind so gewählt, daß eine gerade Permutation von (1234) herauskommt, wenn man die fehlende Ziffer dahinterschreibt.
Die Komponenten der Wellenfunktion, deren jede wieder ein Vektor aus 4 Komponenten ist, seien der Reihe nach mit

$$
\psi_{14}, \psi_{24}, \psi_{34}, \psi_{23}, \psi_{31}, \psi_{12}, \psi_{1}, \psi_{2}, \psi_{3}, \psi_{4}
$$

bezeichnet und noch $\psi_{\lambda_{\mu}}=-\psi_{\mu \lambda}$ gesetzt. Dann bekommen die Gln. (VI.1) die Gestalt

$$
\begin{aligned}
& \left(a_{\nu} \partial_{\nu}+3 \%\right) \psi_{\lambda_{\mu}}+2\left(\partial_{\lambda} \psi_{\mu}-\partial_{\mu} \psi_{\lambda}\right)=0 \\
& (\lambda, \mu=1, \ldots, 4 ; \lambda \neq \mu) \\
& \left(a_{\nu} \partial_{\nu}+3 \varkappa\right) \psi_{\lambda}+2 \partial_{v^{\prime}} \psi_{v^{\prime} \lambda^{\prime}}=0 \\
& \left(\lambda=1, \ldots, 4 ; v^{\prime} \neq \lambda\right)
\end{aligned}
$$

das sind $6+4=10$ Gleichungen.

Diesmal transformieren wir mit der Matrix

$$
\left.\begin{array}{ccccc}
0 & 0 & 0 & 0 & 0 \\
0 & 0 & 0 & 0 & 0 \\
0 & 0 & 0 & 0 & 0 \\
0 & 0 & 0 & 0 & 0 \\
0 & 0 & 0 & 0 & 0 \\
c_{3} c_{4} & 0 & 0 & 0 & 0 \\
0 & c_{2} c_{4} c_{3} & 0 & 0 & 0 \\
0 & 0 & c_{3} c_{4} c_{1} & 0 & 0 \\
0 & 0 & 0 & a_{1} c_{4} c_{2} & 0 \\
0 & 0 & 0 & 0 & c_{1} c_{2} c_{3}
\end{array}\right)
$$

aufgespannt. Auf dieselbe Weise wie vorhin erhält man daraus durch die Bedingung des Senkrechtstehens die Beziehungen

$$
\begin{aligned}
& \psi_{14}^{\prime}+\psi_{24}^{\prime}+\psi_{34}^{\prime}+\psi_{23}^{\prime}+\psi_{31}^{\prime}+\psi_{12}^{\prime} \\
& \text { und } \\
& {\psi^{\prime}}_{14}^{\prime}+\psi^{\prime}{ }_{31}+\psi_{12}^{\prime}+\psi_{2}^{\prime}+\psi_{3}^{\prime}+\psi_{4}^{\prime}=\mathbf{0} \text {, } \\
& \psi_{24}^{\prime}+\psi_{23}^{\prime}+\psi^{\prime}{ }_{12}+\psi_{1}^{\prime}+\psi_{3}^{\prime}+\psi_{4}^{\prime}=0 \text {, } \\
& \psi_{34}^{\prime}+\psi_{23}^{\prime}+\psi_{31}^{\prime}+\psi_{1}^{\prime}+\psi_{2}^{\prime}+\psi_{{ }^{\prime}}^{\prime}=0 \text {, } \\
& \psi^{\prime}{ }_{14}+\psi_{24}^{\prime}+\psi_{34}^{\prime}+\psi_{1}^{\prime}+\psi_{2}^{\prime}+\psi_{3}^{\prime}=0 \text {, }
\end{aligned}
$$$$
-\psi^{\prime}{ }_{1}-\psi_{2}^{\prime}-\psi^{\prime}{ }_{3}-\psi^{\prime}{ }_{4}=0
$$

die sich leicht vereinfachen lassen. Addition der 4 Gln. (VI. 8) ergibt $2 \sum_{\mu, r^{\prime}} \psi_{\mu \nu}^{\prime}=-\sum_{\lambda} \psi_{i}^{\prime}$; hieraus und aus $\sum_{\mu, v} \psi_{\mu \nu}^{\prime}=\sum_{\lambda} \psi_{\lambda}^{\prime}$ folgt $\sum_{\mu, v} \psi_{\mu \nu}^{\prime}=\sum_{\lambda} \psi_{\lambda}^{\prime}=0$. Die erste (VI. 8) heißt jetzt $\psi_{1}^{\prime}=\psi_{14}^{\prime}+\psi_{31}^{\prime}+\psi_{12}^{\prime}$; transformiert man wieder auf die ungestrichenen Größen, indem man von links mit $\alpha_{3} \alpha_{4} \alpha_{2}$ multipliziert, so kommt $\psi_{1}=\alpha_{4} \psi_{41}+\alpha_{3} \psi_{31}+\alpha_{2} \psi_{21}$, und so wird allgemein $\psi_{\lambda}=\alpha_{v} \psi_{v}$. Dies setzen wir in die erste (VI. 7) ein und erhalten 
$\left(a_{\nu} \partial_{\nu}+3 \varkappa\right) \psi_{\lambda \mu}+2 \alpha_{\nu}\left(\partial_{\lambda} \psi_{\nu \mu}-\partial_{\mu} \psi_{\nu} \hat{\lambda}\right)=0$.

Wie früher ist dann die zweite (VI.7) von selbst erfüllt. (VI.9) ist das gesuchte System von Wellengleichungen zusammen mit den Nebenbedingungen

$\psi_{\lambda_{\mu}}+\psi_{\mu \lambda}=0$ und $\quad a_{\lambda}{ }^{c}{ }_{\mu} \psi_{\lambda \mu}=0$,

von denen die zweite aus $\sum_{\lambda, \mu} \psi^{\prime} \lambda_{\mu}=0$ folgt, wenn man auf $\psi_{\lambda_{\mu}}$ umrechnet und noch mit $\alpha_{1} \alpha_{2} \alpha_{3} \alpha_{4}$ multipliziert.

VII. Zuordnung von Masse und Spin

für die in den Wellengleichungen enthaltenen Teilchen (f ür $N=3$ )

Die physikalischen Eigenschaften, welche ein elementares Teilchen kennzeichnen, sind außer der Ladung zunächst Masse und Spin; hinzu kommt, obwohl empirisch nur in wenigen Fällen bekannt (für Elektron, Proton und Neutron), das magnetische Moment. Es handle sich zunächst um die Zuordnung von Massen- und Spinwerten gemäß den Wellengleichungen (VI.6) und (VI.9), (VI. 10).

An Stelle der Wellengleichung (VI.6) benutzt man hier am bequemsten die Gl.

$$
\left(\gamma_{\mu} \partial_{\mu}+3 \varkappa\right) \psi=0
$$

wo $\gamma_{\mu}$ die Bedeutung (V.2) haben soll (im vorigen Abschnitt $\gamma_{\mu}^{\prime}$ ). Das zugehörige Quadrat des Impulsmoments (V.6) ist

$$
\mathrm{S}^{2}=\frac{1}{4}\left(\begin{array}{rrrr}
11 & -4 & -4 & 0 \\
-4 & 11 & -4 & 0 \\
-4 & -4 & 11 & 0 \\
0 & 0 & 0 & 3
\end{array}\right) \times 1
$$

$S^{2}$ ist nicht mit allen $\gamma_{\mu}$ vertauschbar, sondern nur mit $\gamma_{4}$. Daraus folgt, daß der Operator der Wellengleichung (VII. 1) nur im Ruhsystem mit $S^{2}$ vertauschbar wird, also nur in diesem Masse und Spin einander eindeutig zugeordnet werden können. Zur Kennzeichnung einer bestimmten Teilchensorte genügt' aber jedenfalls die Angabe des Spinmoments in einem ausgezeichneten Koordinatensystem wie dem Ruhsystem. Hier nimmt (VII. 1) die vereinfachte Form

$$
\left(\gamma_{4} \partial_{4}+3 \varkappa\right) \psi=0
$$

19 Durch die Doppelindizierung $\psi_{\lambda, \zeta}(\lambda=-1, \ldots, 4$; $\zeta=1, \ldots, 4)$ soll die 4.4-reihige Darstellung zum Ausdruck gebracht werden, wobei die ausgeschriebenen Matrizen auf $\lambda$, die $\alpha_{\mu}$ auf $\zeta$ wirken. an, und mit dem Ansatz $\psi=\psi_{\lambda, \zeta}=a_{\lambda, \zeta} e^{\varepsilon x_{4}} \operatorname{wird}^{19}$

$$
\left(-\varepsilon \gamma_{4}+3 \varkappa\right) \psi=0 \text {. }
$$

Die möglichen Eigenwerte von $\varepsilon$ sind somit

$$
\varepsilon^{\prime}=\frac{E_{0}}{\hbar c}= \pm \frac{3 \%}{\left|\gamma^{\prime}{ }_{4}\right|}\left(E_{0} \text { Ruhenergie }\right) .
$$

Mit (VII. 3a) kann $S^{2} \psi=i \psi$ simultan befriedigt werden $\left[\lambda=\sigma(\sigma+1)\right.$ ist Eigenwert von $\hat{S}^{2}$ und $\sigma$ Spinquantenzahl]. Nun ist hier

$$
\gamma_{4}=\left(\begin{array}{rrrr}
-1 & 0 & 0 & 0 \\
0 & -1 & 0 & 0 \\
0 & 0 & -1 & 0 \\
2 & 2 & 2 & 3
\end{array}\right) \times c_{4} .
$$

Man kann sich jeweils auf den ersten KroneckerFaktor beschränken und bestätigt leicht die gemeinsamen Eigenvektoren von (VII. 2) und (VII. 5)

$$
\begin{aligned}
& \left(\begin{array}{llll}
0, & 0, & 0, & 1
\end{array}\right) \\
& (1, \quad 1, \quad 1,-3 / 2) \\
& (1,-1, \quad 0, \quad 0) \\
& (1, \quad 0,-1, \quad 0)
\end{aligned}
$$

welche der Reihe nach zu den Eigenwerten gehören:

$$
\begin{array}{lr|r|rr}
\varepsilon^{\prime}: & \pm \varkappa & \pm 3 \% & \pm 3 \% \pm 3 \% \\
\lambda: & 3 / 4 & 3 / 4 & 15 / 4 & 15 / 4 .
\end{array}
$$

Es ergibt sich somit, daß durch die Wellengleichung (VI.6) bzw. (VII.1) das Vorhandensein von drei verschiedenen Teilchen zum Ausdruck gebracht wird, von denen zwei dieselben Spinwerte $\sigma=1 / 2$, aber verschiedene Massen $\varkappa \hbar / c$ und $3 \varkappa \hbar / c$ besitzen, das dritte den Spin $\sigma=3 / 2$ und die größere Masse $3 \varkappa \hbar / c^{20}$. Das Verhältnis der möglichen Massenwerte ist also $1: 3$.

Der Umstand, daß dieselbe (irreduzible) Wellengleichung Teilchen mit verschiedenen individuellen Eigenschaften enthält, bringt besonders deutlich zum Ausdruck, daß verschiedene Teilchen als „Anregungsstufen" desselben Gebildes aufgefaßt werden können (wobei „Entartungen“, wie im vorliegenden Falle, nicht ausgeschlossen sind) und daß diese Teilchen eine gewisse innere Abhängigkeit voneinander aufweisen. Letzteres wird bedeutsam bei Anwesenheit von äußeren Feldern, wo sich die Eigenschaften der Teilchen gewissermaßen durchmischen und eine eindeutige Zuordnung von Spinwerten, magnetischen

$20 \mathrm{Da}$ im Falle des dritten Teilchens doppelt so viele linear unabhängige Eigenvektoren wie für die beiden ersten auftreten, entspricht natürlich der doppelten Anzahl möglicher Einstellungen des Spins $3 / 2$ gegenüber $1 / 2$ in einem äußeren Felde. 
Momenten und Massenwerten im allgemeinen nicht mehr möglich sein wird ${ }^{21}$.

Eine analoge Betrachtung führt im Falle der Wellengleichung (VI.9) (5•4-reihige Darstellung) zu den simultanen Eigenwerten ${ }^{22}$

$$
\begin{array}{l|r|r}
\varepsilon^{\prime}: \pm \varkappa^{\prime} & \pm 3 \varkappa^{\prime} & \pm 3 \varkappa^{\prime} \\
\lambda: 15 / 4 & 3 / 4 & 15 / 4
\end{array}
$$

Es ergeben sich wiederum drei verschiedene Teilchen: Hier besitzt das Teilchen der kleinen Masse $x^{\prime} \hbar / c$ den Spin $3 / 2$, dagegen existieren zwei Modifikationen von Teilchen mit der größeren Masse $3 x^{\prime} \hbar / c$ mit den Spinwerten $1 / 2$ und $3 / 2$. Da kein Anlaß zu der Annahme besteht, daß die $x$-Werte in den verschiedenen irreduziblen Darstellungen dieselben sind ${ }^{23}$, so sind die $x$-Werte in Tab. (VII. 8) durch einen Akzent gekennzeichnet. Wiederum ist das Massenverhältnis $1: 3^{24}$.

\section{Berechnung des magnetischen Moments}

Ähnlich den Verhältnissen beim Dirac-Elektron wird den in den Wellengleichungen (VI.6), (VI.9) und (VI.10) enthaltenen Teilchen je ein bestimmtes magnetisches Moment zukommen. Dieses ergibt sich aus der der Feldstärke proportionalen Energiestörung, wenn das System einem äußeren (statischen) Magnetfelde $\mathfrak{S}$ ausgesetzt wird. Man wird einem äußeren Felde Rechnung tragen, indem man in den Wellengleichungen den Energie-Impulsoperator $\partial_{\mu}$ durch

$$
\partial_{\mu}{ }^{\prime}=\partial_{\mu}+i e \varphi_{\mu} ; \quad \varphi_{\mu}=\frac{1}{\hbar c}(\mathfrak{A}, i \varphi)
$$

( $\mathfrak{A}, i \varphi$ elektromagnet. Viererpotential) ersetzt. Für unsere Zwecke setzen wir ein statisches Magnetfeld $\mathfrak{S}=\operatorname{rot} \mathfrak{A}$ und somit

$$
\partial_{k}^{\prime}=\partial_{k}+i e \varphi_{k}(k=1,2,3), \partial^{\prime}{ }_{4}=\partial_{4}
$$

voraus.

Wir erläutern das für die Berechnung des magnetischen Moments erforderliche Störungsverfahren hier nur an der erweiterten Wellengleichung (VI.6)

$$
\left(c_{\mu} \partial^{\prime} \mu+3 \%\right) \psi_{i}+2 \partial^{\prime} i_{\nu}\left(\iota_{\mu} \psi_{\mu}\right)=0,
$$

und zwar für das Teilchen mit $\sigma=1 / 2$ und der klei-

21 S. auch Diss. von A. Hueck, Freiburg 1949: Über die Klassifikation von freien Elementarteilchen mit beliebiger Spinquantenzahl auf Grund ihres Verhaltens in bewegten Bezugssystemen.

22. In der Tabelle ist von einer Verdoppelung der Eigenzustände für die Spinwerte $3 / 2$ abgesehen worden. nen Masse $\varkappa \hbar / c^{25}$. Das Gleichungssystem (VIII. 3) lautet mit dem Ansatz $\psi_{\mu}=u_{\mu} e^{-\varepsilon x_{4}}$ ausführlich:

$$
\begin{gathered}
\left(c_{1} \partial_{1}^{\prime}+c_{2} \partial_{2}^{\prime}+c_{3} \partial_{3}^{\prime}-a_{4} \varepsilon+3 \%\right) \psi_{k} \\
+2 \partial_{k}\left(c_{1} \psi_{1}+c_{2} \psi_{2}+a_{3} \psi_{3}+c_{4} \psi_{4}\right)=0, \\
(k=1,2,3) \\
\text { (VIII. 3a-c) } \\
\left(c_{1} \partial_{1}^{\prime}+a_{2} \partial_{2}^{\prime}+a_{3} \partial_{3}^{\prime}-a_{4} \varepsilon+3 \varkappa\right) \psi_{4} \\
-2 \varepsilon\left(c_{1} \psi_{1}+c_{2} \psi_{2}+\alpha_{3} \psi_{3}+c_{4} \psi_{4}\right)=0 .
\end{gathered}
$$

Wir machen den Störungsansatz

(VIII. 3d)

$$
\begin{aligned}
\psi_{\mu}=\psi_{\mu} \mu^{(0)}+\psi_{\mu}^{(1)}+\psi_{\mu}^{(2)} & +\ldots, \\
\varepsilon & =\varepsilon^{(0)}+\varepsilon^{(1)}+\varepsilon^{(2)}+\ldots
\end{aligned}
$$

und setzen in nullter Näherung $\partial^{\prime}{ }_{k}=0$ (ruhendes Teilchen ohne äußeres Feld). Auch weiterhin soll der Einfluß von $\partial^{\prime}{ }_{k}$ als klein, d. h. als Störung aufgefaßt werden. Für das genannte Teilchen gilt also in nullter Näherung [erste Zeile von (VII.6)]:

$$
\psi_{1}{ }^{(0)}=\psi_{2}{ }^{(0)}=\psi_{3}{ }^{(0)}=0, \quad\left(-\varepsilon^{(0)} a_{4}+\varkappa\right) \psi_{4}{ }^{(0)}=0 .
$$

Entscheiden wir uns für positive Energie, so wird $\varepsilon^{(0)}=+\varkappa$ und somit

$$
\left(-c_{4}+1\right) \psi_{4}{ }^{(0)}=0 .
$$

Für die zu(VIII. 3a) adjungierte Wellenfunktion nullter Näherung $\bar{\psi}_{4}{ }^{(0)}$ gilt $\bar{\psi}_{4}{ }^{(0)}\left(-\alpha_{4} \partial_{4}+\varkappa\right)=0$ und daher entsprechend dem Zeitansatz $\bar{\psi}_{4}{ }^{(0)}=\bar{u}_{4}{ }^{(0)} e^{+\varepsilon x_{4}}$

$$
\bar{\psi}_{4}{ }^{(0)}\left(-\epsilon_{4}+1\right)=0 \text {. }
$$

In erster Näherung wird

$$
\left(-\iota_{4}+3\right) \% \psi_{k}^{(1)}=-2 \iota_{4} \partial_{k}{ }^{\prime} \psi_{4}{ }^{(0)}, \quad(k=1,2,3)
$$

$3\left(-c_{4}+1\right) * \psi_{4}^{(1)}=\left(3 c_{4} \varepsilon^{(1)}-d^{\prime}\right) \psi_{4}^{(0)}$

$$
+2 \%\left(\iota_{1} \psi_{1}^{(1)}+c_{2} \psi_{2}^{(1)}+\iota_{3} \psi_{3}^{(1)}\right)
$$

mit der vorübergehenden Abkürzung

$$
\partial^{\prime}=\iota_{1} \partial_{1}{ }^{\prime}+\iota_{2} \partial_{2}{ }^{\prime}+c_{3} \partial_{3}{ }^{\prime},
$$

und wegen (VIII. 4 a) (Verschwinden der einen Hälfte der Wellenfunktionen $\psi_{4, \xi}^{(0)}$ ) nach (VIII. $5 \mathrm{a}-\mathrm{c}$ )

$$
\psi_{k}^{(1)}=-\frac{1}{\%} \partial_{k}^{\prime} \psi_{4}^{(0)}, \quad(k=1,2,3) .
$$

23 Vgl. H. H ö n l, Z. Naturforschg. 2a, 537 [1947].

24 Auf das Massenverhältnis 1:3 haben schon $\mathrm{K} \mathrm{ra}$ mers, Belinfante u. Lubański5 hingewiesen.

25 Dieser Fall hat deswegen besonderes Interesse, weil man in dem Teilchen das Proton vermuten könnte (siehe Schlußabschn. IX). 
Einsetzen in (VIII. 5d) ergibt

$$
\left(-c_{4}+1\right) \psi_{4}^{(1)}=\frac{1}{\%}\left(c_{4} \varepsilon^{(1)}-\partial^{\prime}\right) \psi_{4}{ }^{(0)} .
$$

Die Forderung der Orthogonalität der rechten Seite zur Lösung nullter Näherung

$$
\int \bar{\psi}_{4}{ }^{(0)} a_{4}\left(a_{4} \varepsilon^{(1)}-\partial^{\prime}\right) \psi_{4}{ }^{(0)} d \tau=0
$$

liefert jetzt mit Rücksicht auf (VIII. 4 a) und (VIII. 4 b) sowie der Normierung $\int \bar{\psi}_{4}{ }^{(0)} \alpha_{4} \psi_{4}{ }^{(0)} d \tau=1: \varepsilon^{(1)}=0$. $\psi_{4}{ }^{(1)}$ läßt sich aufbauen aus den vier Eigenfunktionen der homogenen Gleichung $\left(-\alpha_{4} \varepsilon+\varkappa\right) \chi=0$. Dabei können die Eigenfunktionen zum positiven Eigenwert $\varepsilon=+\varkappa$ unberücksichtigt bleiben $\left(\alpha_{4}\right.$ sei diagonal $)$, da sie für die Erfüllung von (VIII.7d) belanglos sind. Somit setzt sich $\psi_{4}{ }^{(1)}$ allein aus den Eigenfunktionen zum Eigenwert $\varepsilon=-\varkappa$ linear zusammen und genügt daher

$$
\left(\iota_{4}+1\right) \psi_{4}^{(1)}=0 .
$$

Erst die zweite Näherung liefert die gesuchte Energiestörung. Man erhält aus (VIII.3):

$$
\begin{aligned}
& \left(-a_{4}+3\right) \varkappa \psi_{k}{ }^{(2)}=-2 \partial_{k}{ }^{\prime}\left(_{4} \psi_{4}{ }^{(1)}\right. \\
& -2 \partial_{k}{ }^{\prime}\left(c_{1} \psi_{1}{ }^{(1)}+c_{2} \psi_{2}{ }^{(1)}+c_{3} \psi_{3}{ }^{(1)}\right)-\partial^{\prime} \psi_{k}^{(1)} \\
& (k=1,2,3) \\
& 3\left(-a_{4}+1\right) \varkappa \psi_{4}{ }^{(2)}=3 a_{4} \varepsilon^{(2)} \psi_{4}{ }^{(0)} \\
& +2 \%\left(a_{1} \psi_{1}{ }^{(2)}+a_{2} \psi_{2}{ }^{(2)}+c_{3} \psi_{3}{ }^{(2)}\right)-\partial^{\prime} \psi_{4}{ }^{(1)} \text {; }
\end{aligned}
$$

weiter aus (VIII.9a-c) mit Rücksicht auf (VIII. $7 \mathrm{a}-\mathrm{c})$ :

$\psi_{k}^{(2)}=-\frac{1+3 a_{4}}{4 \%} \partial_{k}{ }^{\prime} \psi_{4}{ }^{(1)}+\frac{1}{4 \varkappa^{2}}\left(\partial^{\prime} \partial_{k}{ }^{\prime}+2 \partial^{\prime}{ }_{k} \partial^{\prime}\right) \psi_{4}{ }^{(0)}$

und

$$
\begin{gathered}
c_{1} \psi_{1}{ }^{(2)}+c_{2} \psi_{2}{ }^{(2)}+c_{3} \psi_{3}{ }^{(2)}=-\frac{1}{4 \%} \partial^{\prime}\left(1+3 \alpha_{4}\right) \psi_{4}{ }^{(1)} \\
+\frac{1}{4 \varkappa^{2}}\left\{\alpha_{1} \partial^{\prime} \partial_{1}{ }^{\prime}+\alpha_{2} \partial^{\prime} \partial_{2}{ }^{\prime}+\alpha_{3} \partial^{\prime} \partial_{3}{ }^{\prime}+2 \partial^{\prime 2}\right\} \psi_{4}{ }^{(0)} .
\end{gathered}
$$

Die endgültige Gleichung für $\psi_{4}^{(2)}$ wird damit, vgl. (VIII. 9d):

$$
3\left(-\alpha_{4}+1\right) \varkappa \psi_{4}{ }^{(2)}=3 \alpha_{4} \varepsilon^{(2)} \psi_{4}{ }^{(0)}+\frac{1}{2 \varkappa}\{\ldots\} \psi_{4}{ }^{(0)},
$$

da der Zusatz auf der rechten Seite $-\frac{3}{2} \partial^{\prime}\left(1+\alpha_{4}\right) \psi_{4}{ }^{(1)}$ mit Rücksicht auf (VIII. 8) wegfällt. Die Anwendung der Orthogonalitätsbedingung für die rechte Seite von (VIII. 11) liefert jetzt wegen (VIII. $4 \mathrm{a}, \mathrm{b}$ )

$$
\varepsilon^{(2)}=-\frac{1}{2 \varkappa} \int{\overline{\psi_{4}}}^{(0)}{ }^{(2}\{\ldots\} \psi_{4} d \tau .
$$

Die Ausrechnung der geschweiften Klammer ergibt nach kurzer Zwischenrechnung, vgl. (VIII.6) und (VIII. 10),

$$
\begin{aligned}
& \{\ldots\}=3[\Delta+2 \text { i } e \overrightarrow{(\phi}, \text { grad })] \\
& \qquad+i e\left[a_{1} a_{2}\left(\frac{\partial \varphi_{2}}{\partial x_{1}}-\frac{\partial \varphi_{1}}{\partial x_{2}}\right)+\text { cycl. }\right]
\end{aligned}
$$

( $\triangle$ Laplacescher Operator, $\operatorname{div} \vec{\varphi}=0$ ). Somit bei Einführung von $\vec{\sigma}=\left(i a_{2} a_{3}, i c_{3} c_{1}, i c_{1} a_{2}\right)$ :

$$
\begin{gathered}
\varepsilon^{(2)}=-\frac{1}{2 \%} \int{\overline{\psi_{4}}}^{(0)} \alpha_{4}\{\Delta+2 i e \overrightarrow{(\vec{\varphi}, \text { grad })} \\
\left.+\frac{e}{3}(\vec{\sigma}, \operatorname{rot} \vec{\varphi})\right\} \psi_{4}{ }^{(0)} d \tau
\end{gathered}
$$

Die beiden ersten Bestandteile von (VIII.12) entsprechen der kinetischen Energie und der Stromwechselwirkung mit dem Magnetfeld. Der letzte Term kann als die Wechselwirkungsenergie eines magnetischen Moments parallel dem Spin vom Betrage

$$
\mu=\frac{1}{3} \frac{e \hbar}{2 m_{0} c}
$$

mit dem äußeren Felde aufgefaßt werden; der Faktor der magnetischen Anomalie des Teilchens ist mit Rücksicht auf den Spin $\hbar / 2$ somit

$$
g=2 / 3 \text {. }
$$

(VIII. 13a)

In analoger Weise lassen sich die g-Faktoren für die andern in den Wellengleichungen zur Stufe $N=3$ enthaltenen Teilchen berechnen; wir geben die $\mathrm{Zu}$ sammenstellung in der Tabelle des Schlußabschnittes ${ }^{26}$.

\section{Ergebnis und Schlußbemerkungen}

Legt man einer Theorie der Elementarteilchen Wellengleichungen vom Diracschen Typus (I.3) zugrunde und konstruiert die darin auftretenden Diracähnlichen Matrizen $\gamma_{\mu}$ gemäß der de Broglieschen Fusionsmethode (I.4), so erhebt sich die rein mathematische Frage nach der vollständigen Ausreduktion

26 Die Verf. danken Hrn. A. W. M a ue herzlich für wertvolle Hilfe bei den Rechnungen. Eine einheitliche Behandlung der magnetischen Momente folgt demnächst in einer Arbeit von Hrn. Maue in Z. Physik. 


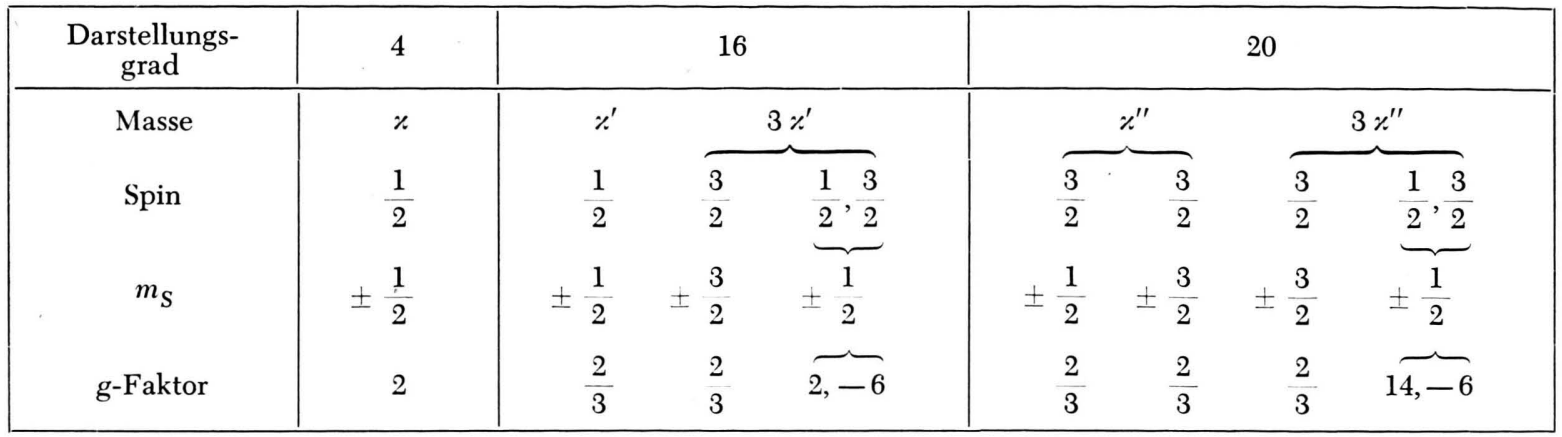

Tab. 1.

der Matrizensysteme (Abschn. II-V) und den zugehörigen ausreduzierten Wellengleichungen (Abschnitt VI). Die Darstellungen der $\gamma_{\mu}$ für die Verschmelzungsstufen $N=1$ und $N=2$ sind nach Dirac und Kemmer bekannt (Elektron bzw. Mesonen mit Spin 0 und $\hbar$ ). In analoger Weise lassen sich die irreduziblen Darstellungen für den Fall $N=3$ angeben. Man gelangt dabei zu 4-, 16- und 20-reihigen Darstellungen, wobei die erste einem Dirac-Teilchen entspricht (also nichts Neues liefert), während die 16und 20-reihigen Darstellungen je 3 Teilchen mit verschiedenen Massen- oder Spinwerten enthalten. Es ist jedoch nicht möglich, jedem dieser Teilchen auch ein bestimmtes magnetisches Moment zuzuordnen. Vielmehr erfolgt bei Anwesenheit eines Magnetfeldes eine teilweise Mischung der beiden Spinwerte $1 / 2$ und $3 / 2$, soweit sie zur gleichen Masse gehören. Ein solches Gemisch besitzt zwar keinen definierten Spin, aber eine bestimmte Komponente des Spins in Feldrichtung (magnetische Quantenzahl $m_{\mathrm{s}}$ ) und ebenso des magnetischen Momentes. Aus ihrem Verhältnis errechnet sich ein g-Faktor, der also hier im Gegensatz zu den gewohnten Verhältnissen von der Einstellung im Magnetfelde abhängt. Die Zuordnung von Massenwerten, Spinwerten, magnetischen Quantenzahlen und Angaben über das magnetische Moment sind der Tab. 1 zu entnehmen (Abschn. VII u. VIII). Bei den Spingemischen sind jeweils beide beteiligten Spinwerte angeführt und durch ein Komma getrennt; es treten jeweils zwei wesentlich verschiedene Gemische mit verschiedenen $g$-Faktoren auf.

Das Verhältnis der Massenkonstanten $\varkappa: \varkappa^{\prime}: \varkappa^{\prime \prime}$ für die verschiedenen Darstellungsgrade bleibt vorläufig unbestimmt und kann durch die vorliegende Theorie ohne zusätzliche Annahmen jedenfalls nicht erfaßt werden. Dagegen ist das Massenverhältnis innerhalb der Gruppen der 16- und 20-reihigen Darstellungen genau 1:3. Zum schwereren Teilchen gehören je die Spinwerte $\hbar / 2$ und $3 \hbar / 2$. Es ist besonders bemerkenswert, daß die verschiedenen Teilchen eines bestimmten Darstellungsgrades nicht unabhängig voneinander sind, sondern im Prinzip gleichzeitig vorhanden sein müssen, da sie als Lösungen derselben Wellengleichungen auftreten; die schwereren Teilchen sind gleichsam „Anregungsstufen“ der leichteren.

Unter den Teilchen der Verschmelzungsstufe $N=3$ befindet sich keines, das dem Proton entspricht, da dessen magnetisches Moment nach I. Rabi etwa 2,79 Kernmagnetonen beträgt (keiner der g-Faktoren zum Spin $\hbar / 2$ liegt in der Nähe des doppelten Betrages hiervon). Es ist denkbar, daß das Proton einer höheren ungeraden Verschmelzungsstufe zuzuordnen wäre. Wahrscheinlicher scheint allerdings, daß ein neuer Gesichtspunkt hinzutreten muß: Proton und Neutron sind nach der Theorie der Kernkräfte als Modifikationen desselben Gebildes aufzufassen und sind außer von einem elektromagnetischen von einem Mesonenfeld umgeben. Diesem Umstand ist aber bei der korrespondenzmäßigen Begründung der Theorie (Bopp) in keiner Weise Rechnung getragen worden. Auch die merkliche Abweichung des g-Wertes von einem einfachen rationalen Zahlenwert weist auf einen in die bisherige Theorie nicht einbezogenen neuen Gesichtspunkt hin.

Die vorstehenden Überlegungen mögen immerhin zeigen, daß sich der de Brogliesche Ansatz zu einer geschlossenen Theorie der Elementarteilchen entwikkeln läßt und daß es lohnend erscheint, diesen Ansatz zu verfolgen. Der ins Einzelne gehende Vergleich mit den noch spärlichen Erfahrungen über die individuellen Eigenschaften der Elementarteilchen scheint indessen noch verfrüht. 